\title{
Réponse des macroinvertébrés benthiques (éphéméroptères, plécoptères, trichoptères) aux pressions anthropiques dans un contexte de changement climatique sur le bassin versant de l'Ourika (Haut-Atlas du Maroc)
}

\author{
Response of benthic macroinvertebrates (ephemeroptera, \\ plecoptera, trichoptera) to anthropogenic pressures in a \\ context of climate change in the Ourika watershed \\ (High Atlas of Morocco)
}

\author{
J.-R. Zuedzang Abessolo*, M. Yacoubi Khebiza, M. Messouli
}

Université Cadi Ayyad, Faculté des Sciences Semlalia Marrakech, Laboratoire eau, biodiversité et changement climatique, associé CNRST, boulevard Prince My Abdellah, BP 2390, 40000 Marrakech, Maroc

zajennyrevany@gmail.com

\begin{abstract}
Résumé - Les changements globaux marqués par une accentuation des pressions anthropiques et climatiques ont un impact sur les écosystèmes lotiques et la faune aquatique. Nous avons analysé leur influence sur l'évolution des peuplements benthiques d'éphéméroptères, de plécoptères et de trichoptères (EPT) au sein du bassin versant de l'Ourika, situé dans le Haut-Atlas du Maroc. L'inventaire des espèces recensées avant et après 2000 révèle que la richesse spécifique a augmenté à l'Oukaïmeden, milieu de haute altitude, tandis qu'elle a diminué à l'Ourika milieu moyenne altitude. À l'Ourika, la richesse spécifique en taxa EPT est passée de 37 avant 2000 à 24 après 2000, soit une baisse de $35 \%$. À l'Oukaïmeden, la richesse spécifique en EPT est passée de 27 avant 2000 à 32 après 2000 , soit une augmentation de $15 \%$. Ces changements résultent essentiellement de la régression de la qualité du milieu aquatique sous la pression anthropique à l'Ourika (eaux usées domestiques, déchets solides, remblais, commerces, dragages...). L'habitat aquatique est dégradé, la pollution organique a augmenté et la qualité biologique de l'eau a connu une forte régression. À l'Oukaïmeden, le milieu est beaucoup moins perturbé en dépit d'une activité pastorale assez importante, et du rejet de déchets solides dans le cours d'eau. Les perturbations sont surtout concentrées au sein d'une station, les autres étant relativement épargnées. L'habitat aquatique est favorable à la faune, la pollution organique est restée relativement faible et la qualité biologique de l'eau bien qu'ayant légèrement régressé demeure favorable pour les peuplements étudiés. L'Oukaïmeden apparaissant comme une zone refuge pour les espèces, le milieu présente un grand intérêt pour la conservation.
\end{abstract}


Mots clés - rivière de montagne, biodiversité, bioindicateurs, écologie, changement à long terme.

\begin{abstract}
Global changes have a significant impact on lotic ecosystems and aquatic wildlife. We analyzed their impact on benthic populations of mayflies, stoneflies and caddisflies (Ephemeroptera-Plecoptera-Trichoptera [EPT]) within the Ourika watershed, located in the High Atlas of Morocco. The inventory of species present in the stations before 2000 , and those found after 2000 show that the species richness increased in Oukaïmeden, whose stations are located at high altitude, while it decreased highly in Ourika whose stations are at medium altitude. In Ourika, the specific richness in EPT fell from 37 before 2000 to 24 after 2000 , a decrease of $35 \%$. The number of mayflies species decreased from 13 species before 2000, to 9 after 2000; that of stoneflies from 7 to 5 , caddisflies from 17 to 10 after 2000. In Oukaïmeden, species richness en EPT increased from 27 before 2000 to 32 after 2000 , an increase of $15 \%$. the number of mayflies species increased from 8 species before 2000, to 12 after 2000; stoneflies from 8 to 10 , caddisflies from 11 to 10 . These changes result from the decline in the quality of the aquatic environment submitted to human pressure in Ourika (domestic wastewater, solid waste, embankment on the banks, shops, dredging...). The aquatic habitat has deteriorated, organic pollution is quite large, biological water quality has declined strongly compared to the period before 2000. In Oukaïmeden, however, the aquatic environment is much less disturbed, disruptions are mostly concentrated within a station, the others being relatively spared. The aquatic habitat is suboptimal, organic pollution has remained low and the biological quality of the water remains good despite a slight decline. The Oukaïmeden appearing as a refuge area for populations of mayflies, stoneflies and caddisflies, the region has great interest in the conservation of species.
\end{abstract}

Keywords - mountain river, biodiversity, bioindicators, ecology, long-term change.

\section{Introduction}

Les changements globaux se traduisent au sein des écosystèmes lotiques par une modification des caractéristiques physicochimiques et hydrologiques des cours d'eau, résultant souvent de l'intensification des activités anthropiques. Cela a un impact direct sur les macroinvertébrés benthiques qui sont de bons indicateurs de la santé des écosystèmes aquatiques (USEPA, 2002), et sont les plus utilisés pour l'évaluer (Hellawell, 1986; Barbour et al., 1999; WFD, 2003). C'est dans ce contexte que nous nous sommes intéressés à l'évolution des peuplements d'éphéméroptères, de plécoptères et de trichoptères, au sein du bassin versant de l'oued Ourika, situé dans le Haut-Atlas du Maroc. II s'agit de la première étude mettant en évidence les changements dans la composition faunistique de ces peuplements au sein de la zone d'étude. Nos travaux ont été menés sur deux cours d'eau, l'oued Ourika et l'oued Oukaïmeden. Le bassin de l'Ourika est un sous-bassin du Tensift, celui de l'Oukaïmeden est un sous-bassin de l'Ourika. Ourika et Oukaïmeden sont des zones rurales où se développent principalement 
l'agriculture, l'élevage et le tourisme avec un afflux touristique très important en été à l'Ourika et, inversement, très important en hiver à l'Oukaïmeden. Des travaux antérieurs menés au sein des localités étudiées ont permis de dresser l'inventaire des espèces de ces 3 ordres (Pihan \& Mohati, 1983; Mohati, 1985; Bouzidi, 1989 ; Thomas et al., 1992 ; Badri, 1993; Talami, 1998; Giudicelli et al., 2000; Errochdi et al., 2014). Plus généralement, l'amélioration de la connaissance des éphéméroptères, plécoptères et trichoptères du Maroc a été possible grâce à plusieurs études (Mosely, 1938; Aubert, 1956; Botosaneanu, 1975; Dakki, 1978, 1982; El Agbani, 1984; Giudicelli \& Dakki, 1984; Badri, 1985; Dakki, 1986; Thomas \& Bouzidi, 1986; Dakki, 1987; Ajakane, 1988; Tayoub, 1989 ;; Ouahsine, 1993; El Alami \& Dakki, 1998; El Alami et al., 2000; Berrahou et al., 2001; Alaoui, 2006; Bonada et al., 2008; Hajji et al., 2012; Hajji et al., 2013; Vinçon et al., 2014; Hajji, 2017). Afin de connaître comment évoluent les peuplements étudiés dans le contexte de changements globaux qui prévaut aujourd'hui, nous avons analysé l'évolution de la richesse spécifique et identifié les changements survenus dans la composition faunistique. Nous avons ensuite déterminé les causes de ces changements en analysant l'évolution du milieu aquatique. Bien qu'il n'existe pas de données pour le suivi de la qualité du milieu aquatique dans la zone d'étude, nous avons pu nous appuyer sur des travaux antérieurs menés au sein des mêmes stations pour mettre en évidence les modifications survenues au sein de l'écosystème. Les changements climatiques, source de pressions supplémentaires sur les écosystèmes étudiés, et leur biodiversité ont également été discutés. Les projections climatiques pour le bassin de l'Ourika prévoient une augmentation progressive de la température pour les prochaines années, ainsi qu'une baisse des précipitations (Ayt Ougougdal etal., 2020). Les espèces montagneuses ont une capacité très réduite de se réfugier à plus hautes altitudes pour fuir le réchauffement des températures, ce qui les rend plus vulnérables au risque de disparition.

\section{Matériel et méthode}

\subsection{Choix des stations}

Les stations prospectées (Fig. 1) ont été choisies pour suivre l'évolution des peuplements et pouvoir comparer les résultats de nos travaux avec ceux de travaux antérieurs menés dans les mêmes stations. Pour l'oued Ourika, 3 stations ont été prospectées. La station OR1 $\left(31^{\circ} 13,347^{\prime} \mathrm{N}\right.$; $007^{\circ} 40,730^{\prime} \mathrm{W}$ ) se trouve tout juste en amont du village de Setti Fatma à $1433 \mathrm{~m}$ d'altitude. La station OR2 $\left(31^{\circ} 15,374^{\prime} \mathrm{N} ; 007^{\circ} 44,606^{\prime} \mathrm{W}\right)$ se situe à la commune rurale d'Aghbalou à $1000 \mathrm{~m}$ d'altitude, en aval de la confluence de l'oued Ourika et l'oued Oukaïmeden. La station OR3 $\left(31^{\circ} 22,523^{\prime} \mathrm{N} ; ; \quad 007^{\circ} 46,843^{\prime} \mathrm{W}\right)$ est située à Tnine Ourika, à $850 \mathrm{~m}$ d'altitude. Pour l'oued Oukaïmeden, 3 stations ont également été prospectées. La station O1 $\left(31^{\circ} 12,094^{\prime} \mathrm{N}\right.$; $007^{\circ} 51,520^{\prime} \mathrm{W}$ ) située en amont du lac de barrage Oukaïmeden à $2611 \mathrm{~m}$ d'altitude. La station $\mathrm{O} 2\left(31^{\circ} 12,709^{\prime} \mathrm{N}\right.$; $007^{\circ} 50,986^{\prime} \mathrm{W}$ ) située à $2550 \mathrm{~m}$ d'altitude en aval du barrage Oukaïmeden. La station O3 $\left(31^{\circ} 13,037^{\prime} \mathrm{N}\right.$; 


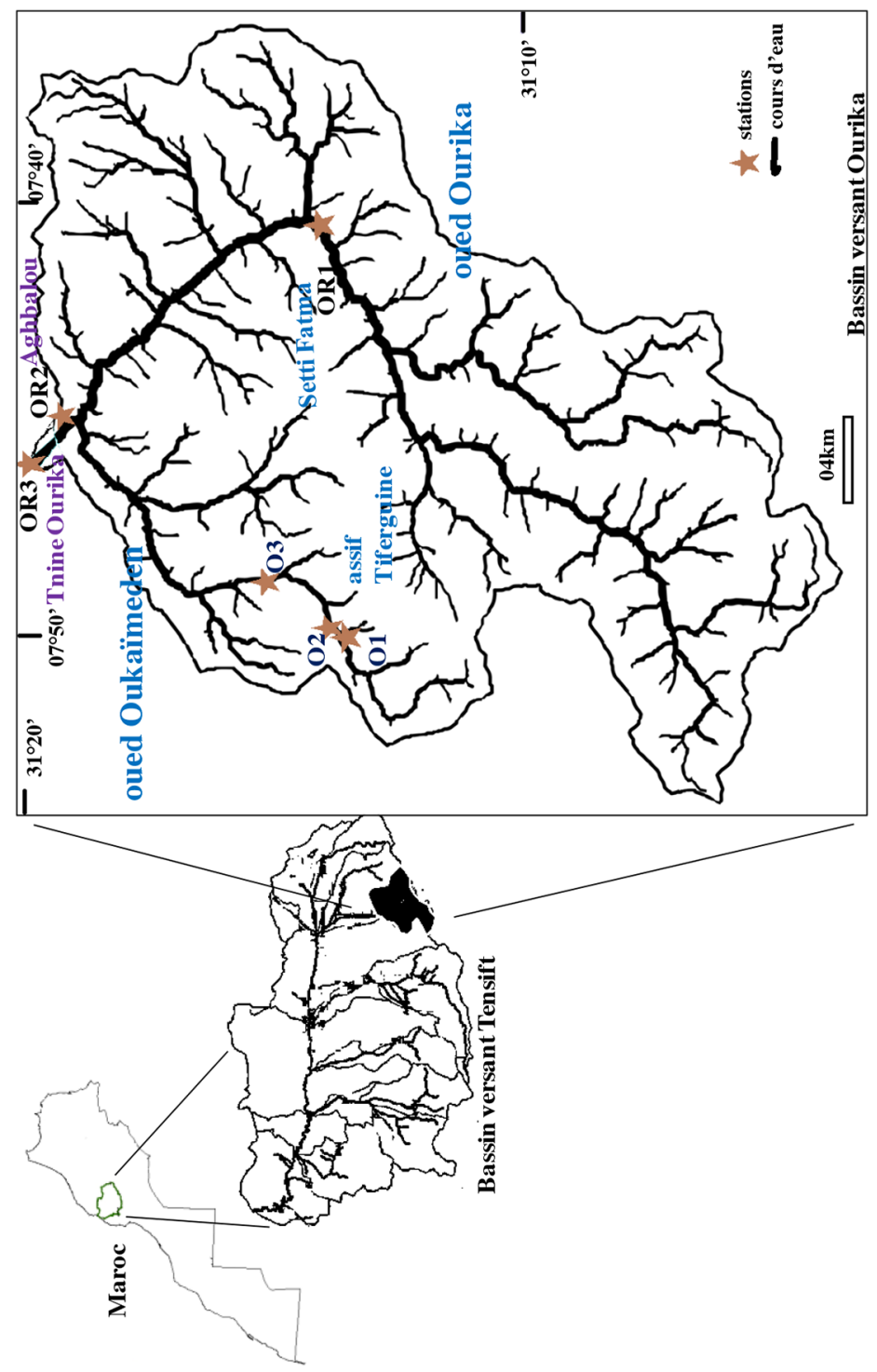


$007^{\circ} 50,376^{\prime} \mathrm{W}$ ) située à $2483 \mathrm{~m}$ d'altitude, environ $300 \mathrm{~m}$ en aval de la confluence avec l'assif Tiferguine.

\subsection{Caractérisation des peuplements de macroinvertébrés benthiques}

\subsection{1 Échantillonnages et identification des taxa}

Les prospections ont été menées entre février 2014 et décembre 2015 pour un total de 12 séries d'échantillonnage (février 2014, mai 2014, janvier 2015, février 2015, mars 2015, mai 2015, juin 2015, juillet 2015, août 2015, octobre 2015, novembre 2015, décembre 2015). En plus des 8 microhabitats du protocole d'échantillonnage de I'IBGN (AFNOR NF T90350), un autre échantillonnage qualitatif a été effectué sur une grande variété de substrat, et de couples vitesse-profondeur afin de recueillir un maximum de diversité de taxa. La faune prélevée à l'aide de filets Surber et troubleau est recueillie dans des bocaux, et fixée dans de l'alcool à $70 \%$. Pour chaque station, des données précises ont été collectées pour l'évaluation du milieu aquatique (voir la sect. 2.3, l'habitat aquatique). Des échantillons d'eau ont également été prélevés pour des analyses chimiques.

Au laboratoire, les taxa ont été identifiés à l'aide de loupes binoculaires et de microscopes. L'identification était uniquement basée sur la morphologie des spécimens, il n'y a pas eu d'analyses génétiques.

\subsubsection{Constitution de l'inventaire des ETP avant et après 2000}

À partir des résultats de nos prospections et des données bibliographiques, nous avons dressé l'inventaire total des éphéméroptères, des plécoptères et des trichoptères recensés au sein des stations prospectées. Pour mettre en évidence les changements survenus dans la composition faunistique (disparition et apparition des espèces), nous avons scindé cet inventaire en deux périodes, la période "avant 2000 » et la période «après 2000 ». Les données faunistiques antérieures à cette étude sont issues de travaux menés autrefois au sein des mêmes stations (Pihan \& Mohati, 1983; Mohati, 1985; Bouzidi, 1989; Thomas et al., 1992; Badri, 1993; Talami, 1998; Giudicelli et al., 2000; Errochdi et al., 2014). Une partie des spécimens récoltés durant ces études a été stockée au laboratoire d'hydrobiologie de la faculté des sciences de Tétouan au Maroc, une autre partie a été stockée au Muséum d'histoire naturelle de Marrakech au Maroc. La faune récoltée durant cette étude a été stockée au laboratoire eau, biodiversité et changement climatique de la faculté des sciences Semlalia Marrakech au Maroc.

\subsection{Analyses chimiques et traitement des données}

Après avoir mis en évidence les changements survenus dans la composition faunistique, nous nous sommes intéressés aux causes de 
Tableau 1. Grille de calcul et classes de qualité de l'IPO.

Table 1. Calculation grid and quality classes of IPO.

\begin{tabular}{lllll}
\hline \multicolumn{5}{c}{ Paramètres } \\
\cline { 2 - 5 } & $\begin{array}{l}\text { DBO 5 } \\
(\mathrm{mg} / \mathrm{L})\end{array}$ & Ammonium $(\mathrm{mg} / \mathrm{L})$ & $\begin{array}{l}\text { Nitrites } \\
(\mu \mathrm{g} / \mathrm{L})\end{array}$ & $\begin{array}{l}\text { Orthophosphates } \\
(\mu \mathrm{g} / \mathrm{L})\end{array}$ \\
\hline Classes & & 5 & 15 \\
5 & 2 & $<0.1$ & $6-10$ & $16-75$ \\
4 & $2-5$ & $0,1-0,9$ & $11-50$ & $76-250$ \\
3 & $5,1-10$ & $1-2,4$ & $51-150$ & $251-900$ \\
2 & $10,1-15$ & $2,5-6,0$ & $>150$ & $>900$ \\
1 & $>15$ & $>6$ & & \\
Classes de I'IPO & Valeur de l'IPO & État de la pollution organique \\
5 & $5,0-4,6$ & Nulle & & \\
4 & $4,5-4,0$ & Faible & & \\
3 & $3,9-3,0$ & Modérée & & \\
2 & $2,9-2,0$ & Forte & & \\
1 & $1,9-1,0$ & Très forte & & \\
\hline
\end{tabular}

ces changements. Nous avons évalué les paramètres abiotiques et nous les avons comparés à ceux des données bibliographiques, pour voir comment les modifications survenues au sein du milieu aquatique ont pu contribuer à faire évoluer la richesse spécifique. Les perturbations abiotiques sont en effet l'un des facteurs dominants qui contribuent à la modification du modèle spatial et temporel de la structure des communautés benthiques dans les écosystèmes lotiques (Scrimgeour et al., 1994). Les paramètres abiotiques analysés sont la température, la pollution organique et l'habitat aquatique. Nous avons également évalué la qualité biologique de l'eau. En ce qui concerne la température de l'eau, elle est étroitement liée à celle de l'air. Tout comme l'ont relevé Badri (1993) et Talami (1998), nous avons constaté lors de nos mesures que la température de l'eau est plus faible en hiver, souvent inférieure à $10^{\circ} \mathrm{C}$, elle augmente au printemps, puis est maximale en été avec des valeurs supérieure à $20^{\circ} \mathrm{C}$, elle redescend ensuite en automne. L'écart thermique entre l'hiver et l'été peu atteindre $20^{\circ} \mathrm{C}$.

\subsubsection{La pollution organique}

Au laboratoire, les échantillons d'eau ont été analysés et les paramètres servant à évaluer la pollution organique ont été mesurés. La pollution organique a été évaluée par l'Indice de pollution organique (IPO) de Leclercq \& Maquet (1987). L'IPO est basé sur la concentration en orthophosphates, en ammonium, en nitrites, et en Demande biochimique en oxygène sur 5 jours (DBO5). Chacun de ces 4 paramètres est subdivisé en 5 classes selon la concentration mesurée (Tab. 1). La valeur de l'IPO est calculée en faisant la moyenne des numéros de classes des 4 paramètres (Tab. 1).

La concentration en nitrites a été mesurée par spectrophotométrie après diazotation à la sulfanilamide en présence de $\mathrm{N}$-(1-Naphthyl) éthylènediamine, selon la norme AFNOR T90-013. 
La concentration en ammonium a été mesurée par spectrophotométrie au bleu d'indophénol selon la norme AFNOR T90-025.

La concentration en orthophosphates a été mesurée par dosage à l'acide ascorbique et au réactif combiné selon la norme AFNOR T90-022. La DBO5 a été mesurée par un $\mathrm{DBO}$ mètre en enceinte thermostatée $\left(20^{\circ} \mathrm{C}\right)$.

\subsubsection{L'habitat aquatique}

L'évaluation de l'habitat aquatique a été faite par l'Indice de qualité de I'habitat (IQH). II est adapté de Barbour et al. (1999) par le ministère du Développement durable, de l'Environnement, de la Faune et des Parcs du Canada (MDDEFP, 2013). II convient pour les cours d'eau peu profonds à substrats grossiers (Moisan \& Pelletier, 2008), comme c'est le cas dans cette étude. L'IQH permet de déterminer l'aptitude d'une rivière à supporter la vie aquatique, de caractériser l'existence et la gravité de la dégradation de l'habitat, et aide à déterminer les sources et les causes de la dégradation de l'habitat.

L'indice évalue l'influence de 10 paramètres: le substrat benthique et la disponibilité des abris, l'ensablement et l'envasement, le régime de vélocité et la profondeur, la sédimentation, le degré de marnage, la modification du cours d'eau, la fréquence des seuils, la stabilité des berges, la protection végétale des berges et la largeur de la bande végétale. Chaque paramètre a une note variant de 0 à 20 , la somme des valeurs de ces notes constitue l'Indice de qualité de l'habitat, qui varie de 0 à 200 (annexe I). L'IQH comprend 4 classes: inférieur à 60 ,
I'habitat aquatique est pauvre; de 60 à 99, l'habitat est marginal ; de 100 à 160, l'habitat est sous-optimal; au delà de 160 , l'habitat est optimal.

\subsubsection{La qualité biologique de l'eau}

La qualité biologique de l'eau a été évaluée par l'Indice biologique global normalisé (IBGN) basé sur la polluosensibilité des macroinvertébrés benthiques. Les macroinvertébrés benthiques sont en effet de bons indicateurs de la santé des écosystèmes aquatiques, grâce à leur capacité à intégrer les effets cumulatifs et synergiques, des perturbations physiques, chimiques et biologiques, dans les cours d'eau. Le principe de I'IBGN est de prélever la macrofaune benthique au niveau d'une station, selon un mode d'échantillonnage standardisé. Le calcul de l'indice ce fait à partir d'un tableau standard (annexe II). L'indice est définit en 5 classes, eau de mauvaise qualité $(\leq 4)$, eau de qualité médiocre (85), eau de qualité moyenne (9-12), eau de bonne qualité (13-16), eau de qualité excellente $(\geq 17)$.

\section{Résultats}

\subsection{Prospections}

Les espèces recensées au cours de nos prospections sont présentées en annexe. La détermination de certains individus jusqu'au niveau spécifique n'a parfois pas été possible en raison du stade juvénile des larves ( $L=$ larve). Les annexes III à $\mathrm{V}$ montrent pour chaque station l'inventaire des espèces recensées. La période avant 2000 est notée «av» et la période après 2000 «ap », la présence d'une espèce est marquée par le symbole «+» et 


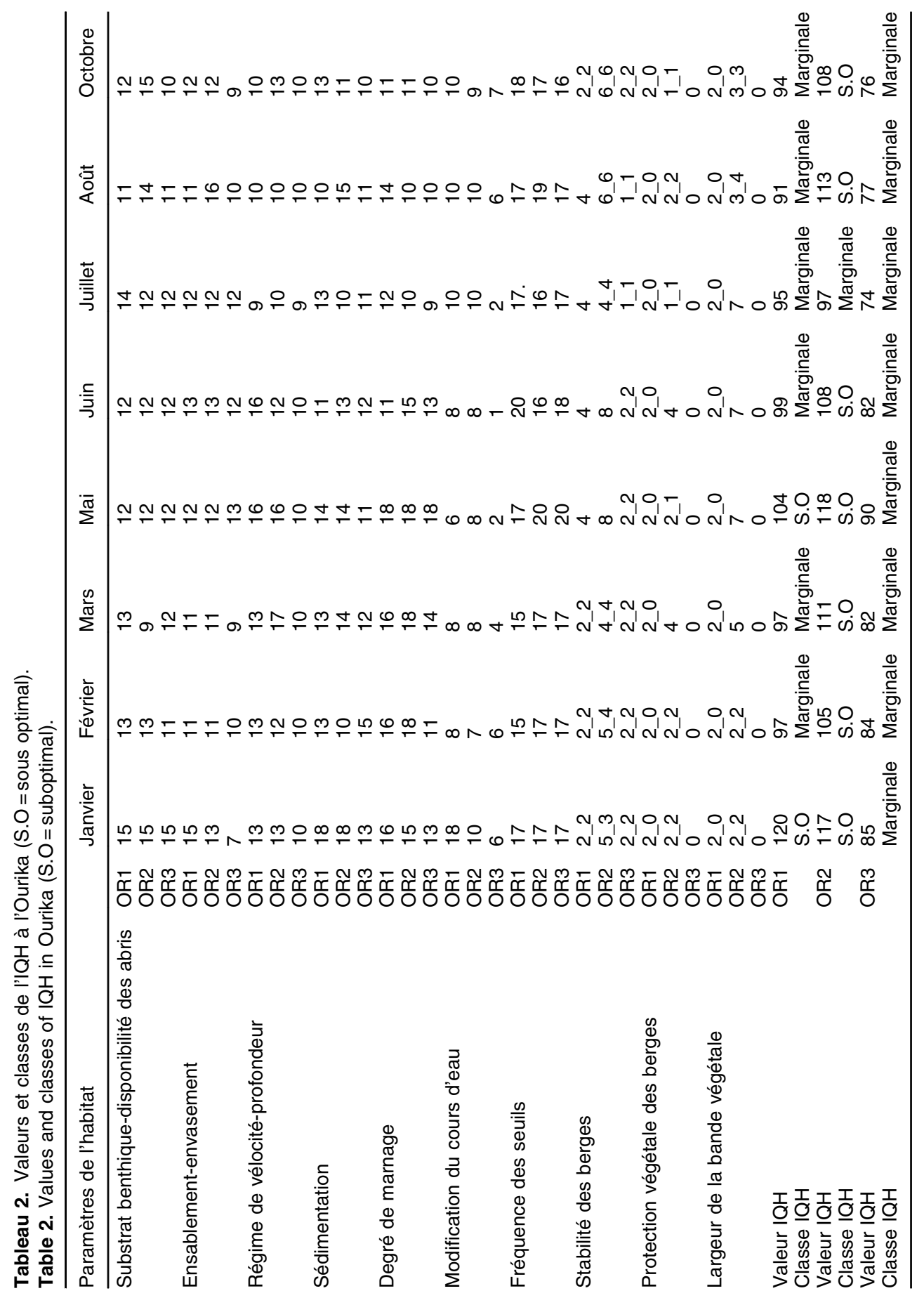


l'absence par «-». Cette étude a permis d'enrichir l'inventaire avec des taxa recensés pour la première fois au sein des stations, ils sont marqués par le symbole ${ }^{*}{ }^{*}$.

\section{2 Évolution de la richesse spécifique}

\subsection{1 Éphéméroptères}

Au total, 19 espèces d'éphéméroptères ont été recensées dans les stations prospectées (annexe III), avec 2 espèces endémiques du Maroc, Alainites oukaimeden (micro-endémique du Haut-Atlas) et Habroleptoides assefae. Cela représente près de $40 \%$ des éphéméroptères du Maroc (48), presqu'autant que pour toute la Tunisie (22) et près de la moitié de ceux d'Algérie (40) (Bebba et al., 2015).

À l'Ourika, la diversité a diminué, 6 espèces ont disparu des stations (A. oukaimeden, O. skoura, O.skhounate, C. dipterum, C. dimorphicum, et Rhithrogena sp.), 2 espèces sont apparues (C. pusilla et Nigrobaetis sp.). La richesse spécifique est passée de 13 espèces recensées avant 2000 , à 9 après 2000 .

À l'Oukaïmeden, la richesse spécifique a augmenté, passant de 8 espèces avant 2000 à 12 après 2000. Le barrage ne semble pas avoir d'effets particuliers sur les peuplements d'éphéméroptères des stations en aval (O2 et O3), dans les 3 stations, la richesse spécifique est à peu près identique. Deux espèces ont disparu des stations (A. oukaimeden et C. luctuosa), 6 sont apparues (A. muticus, H.assafae, Habrophlebia sp., Nigrobaetis sp., B. pavidus et Choroterpes sp.).
Le genre Nigrobaetis est recensé pour la première fois au sein des deux cours d'eau étudiés. Les individus recensés ont une assez bonne tolérance à la pollution organique et semble résister aux perturbations. Dans chaque cours d'eau, ils ont été recensés dans les stations où la qualité biologique de l'eau et la plus dégradée. Cette faculté de pouvoir tolérer les milieux perturbés semble avoir favorisé son expansion dans la zone d'étude. Ailleurs au Maroc, le genre Nigrobaetis est présent avec Nigrobaetis rhithralis (Soldán \& Thomas, 1983), une espèce endémique maghrébine recensée dans le Rif sur l'Oued Tisgris et sur l'Oued Sidi Brahim Ben Arrif dans des stations situées respectivement à $580 \mathrm{~m}$ et $500 \mathrm{~m}$ (El Alami, 2002). L'aire de répartition de cette endémique algérienne s'est d'abord étendue jusqu'au Nord-Ouest du Maroc où les premiers recensements datent de 1998 (El Alami et al., 2000). La diversité des éphéméroptères est donc désormais plus importante dans les stations de haute altitude (Oukaïmeden), tandis qu'elle a considérablement baissé dans les stations de moyenne altitude (Ourika).

\subsubsection{Plécoptères}

Au total, 13 espèces de plécoptères, ont été recensées dans les stations prospectées (Tab. 4), soit $46 \%$ des 28 que compte le Maroc (Errochdi et al., 2014). Parmi ces 13 espèces, 3 sont endémiques du Maroc (S. lepineyi, $C$. atlasica et $A$. chiffensis) avec $A$. chiffensis micro-endémique du Haut-Atlas.

La richesse spécifique des plécoptères a diminuée à l'Ourika, 7 espèces étaient recensées avant 2000 contre 5 
Tableau 3. Valeurs et classes de l'IQH à l'Oukaïmeden $(S . O=$ sous optimal).

Table 3. Values and classes of IQH in Oukaïmeden (S.O=suboptimal).

\begin{tabular}{|c|c|c|c|c|c|c|c|c|c|}
\hline Paramètres de l'habitat & & Janvier & Février & Mars & Mai & Juin & Juillet & Août & Novembre \\
\hline \multirow{3}{*}{$\begin{array}{l}\text { Substrat benthique- } \\
\text { disponibilité des abris }\end{array}$} & $\mathrm{O} 1$ & 17 & 17 & 18 & 17 & 16 & 17 & 17 & 16 \\
\hline & $\mathrm{O} 2$ & 11 & 10 & 10 & 15 & 10 & 10 & 10 & 13 \\
\hline & O3 & 16 & 16 & 15 & 18 & 18 & 16 & 18 & 15 \\
\hline \multirow[t]{3}{*}{ Ensablement-envasement } & 01 & 16 & 16 & 17 & 18 & 16 & 13 & 17 & 17 \\
\hline & O2 & 15 & 15 & 13 & 7 & 10 & 11 & 11 & 11 \\
\hline & O3 & 16 & 16 & 17 & 18 & 12 & 14 & 17 & 13 \\
\hline \multirow{3}{*}{ Régime de vélocité-profondeur } & $\mathrm{O} 1$ & 10 & 9 & 10 & 10 & 7 & 6 & 8 & 10 \\
\hline & $\mathrm{O} 2$ & 8 & 10 & 10 & 8 & 10 & 9 & 8 & 10 \\
\hline & O3 & 10 & 10 & 10 & 10 & 7 & 9 & 10 & 10 \\
\hline \multirow[t]{3}{*}{ Sédimentation } & O1 & 15 & 15 & 15 & 17 & 15 & 17 & 13 & 13 \\
\hline & O2 & 13 & 14 & 15 & 8 & 10 & 15 & 12 & 14 \\
\hline & O3 & 12 & 9 & 17 & 17 & 15 & 11 & 14 & 12 \\
\hline \multirow[t]{3}{*}{ Degré de marnage } & O1 & 10 & 10 & 16 & 12 & 15 & 14 & 10 & 9 \\
\hline & O2 & 13 & 13 & 13 & 15 & 11 & 12 & 14 & 10 \\
\hline & O3 & 15 & 15 & 15 & 17 & 15 & 11 & 11 & 11 \\
\hline \multirow[t]{3}{*}{ Modification du cours d'eau } & O1 & 17 & 17 & 18 & 10 & 10 & 12 & 10 & 13 \\
\hline & $\mathrm{O} 2$ & 18 & 18 & 17 & 18 & 18 & 16 & 18 & 19 \\
\hline & O3 & 17 & 17 & 19 & 18 & 19 & 17 & 18 & 19 \\
\hline \multirow[t]{3}{*}{ Fréquence des seuils } & O1 & 17 & 17 & 17 & 17 & 16 & 18 & 17 & 19 \\
\hline & O2 & 17 & 17 & 17 & 16 & 17 & 18 & 17 & 18 \\
\hline & O3 & 17 & 17 & 17 & 17 & 17 & 18 & 19 & 17 \\
\hline \multirow[t]{3}{*}{ Stabilité des berges } & O1 & 6_6 & 6_6 & 6_5 & 6_6 & 6_5 & 6_6 & $5 \_6$ & 5_5 \\
\hline & $\mathrm{O} 2$ & 6_9 & 6_9 & 9_9 & 9_9 & 4_7 & 3_2 & 5_7 & 7_8 \\
\hline & O3 & 6_6 & $6 \_6$ & 7_7 & 7_7 & $6 \_8$ & 6_6 & $6 \_6$ & 5_6 \\
\hline \multirow[t]{3}{*}{ Protection végétale des berges } & O1 & 2_2 & 2_2 & 2_2 & 4_4 & 5_5 & 7_6 & 4_4 & 3_4 \\
\hline & $\mathrm{O} 2$ & $3 \_3$ & 3_3 & 2 & $6 \_6$ & $2 \_5$ & 2_3 & 1_3 & $2 \_3$ \\
\hline & O3 & 2_2 & 2_2 & 2_3 & 5_6 & 6_6 & 6_6 & 4_4 & 2_3 \\
\hline \multirow[t]{3}{*}{ Largeur de la bande végétale } & O1 & 2_2 & 2_2 & $6 \_3$ & 5_5 & $6 \_5$ & 6_6 & 4_3 & 5_5 \\
\hline & O2 & 1_3 & $1 \_3$ & 1_1 & 1_3 & 1_4 & 1_3 & 0_2 & 1_3 \\
\hline & O3 & $1 \_1$ & $1 \_1$ & 1_3 & 5_5 & 5_5 & 6_9 & $5 \_5$ & 1_5 \\
\hline Valeur IQH & O1 & 122 & 121 & 135 & 131 & 127 & 134 & 118 & 124 \\
\hline Classe IQH & & S.O & S.O & S.O & S.O & S.O & S.O & S.O & S.O \\
\hline Valeur IQH & $\mathrm{O} 2$ & 120 & 122 & 120 & 121 & 109 & 105 & 108 & 119 \\
\hline Classe IQH & & S.O & S.O & S.O & S.O & S.O & S.O & S.O & S.O \\
\hline Valeur IQH & $\mathrm{O} 3$ & 121 & 120 & 160 & 150 & 139 & 135 & 137 & 119 \\
\hline Classe IQH & & S.O & S.O & S.O & S.O & S.O & S.O & S.O & S.O \\
\hline
\end{tabular}

après 2000. Quatre espèces ont disparu des stations (A. chiffensis, L. maroccana, E. ochracea, A. lecerfi), 2 sont nouvelles $(H$. flaviventris et $S$. lepineyi). En examinant les stations individuellement, nous constatons qu'il y a une diminution de la richesse spécifique à la station OR1, et une disparition complète des plécoptères des stations OR2 et OR3.
À l'Oukaïmeden, la richesse spécifique a augmenté, au total 8 espèces $y$ étaient recensées avant 2000 , contre 10 pour la période après 2000 . II y a une grande variabilité de la richesse spécifique des plécoptères d'une station à l'autre semblant être liée au barrage. Entre $\mathrm{O} 1$ en amont du barrage et $\mathrm{O} 2$ en aval, il y a une forte diminution de la richesse spécifique. Lorsqu'on 
Tableau 4. Indice de Pollution Organique (IPO) à l'Ourika.

Table 4. Organic Pollution Index (IPO) in Ourika.

\begin{tabular}{|c|c|c|c|c|c|c|c|c|}
\hline & & DBO5 (valeurs en mg/L) & & & & & & \\
\hline & & Janvier & Février & Mars & Mai & Juin & Août & Octobre \\
\hline \multirow[t]{2}{*}{ OR1 } & Valeur & 12 & 14 & 5 & 5 & 6 & 5,77 & 4 \\
\hline & Classe & 2 & 2 & 4 & 4 & 3 & 3 & 4 \\
\hline \multirow[t]{2}{*}{ OR2 } & Valeur & 17 & 22 & 20 & 30 & 45 & 35 & 22 \\
\hline & Classe & 1 & 1 & 1 & 1 & 1 & 1 & 1 \\
\hline \multirow[t]{3}{*}{ OR3 } & Valeur & 33 & 38 & 30 & 39 & 55 & 62 & 40 \\
\hline & Classe & $\begin{array}{l}1 \\
\text { Ammonium } \\
\text { (valeurs en } \mathrm{mg} / \mathrm{L} \text { ) }\end{array}$ & 1 & 1 & 1 & 1 & 1 & 1 \\
\hline & & Janvier & Février & Mars & Mai & Juin & Août & Octobre \\
\hline OR1 & $\begin{array}{l}\text { Valeur } \\
\text { Classe }\end{array}$ & $\begin{array}{l}0,09 \\
5\end{array}$ & $\begin{array}{l}0,1 \\
4\end{array}$ & $\begin{array}{l}0,04 \\
5\end{array}$ & $\begin{array}{l}0,156 \\
4\end{array}$ & $\begin{array}{l}0,060 \\
5\end{array}$ & $\begin{array}{l}0,03 \\
5\end{array}$ & $\begin{array}{l}0,05 \\
5\end{array}$ \\
\hline OR2 & $\begin{array}{l}\text { Valeur } \\
\text { Classe }\end{array}$ & $\begin{array}{l}0,07 \\
5\end{array}$ & $\begin{array}{l}0,128 \\
4\end{array}$ & $\begin{array}{l}0,08 \\
5\end{array}$ & $\begin{array}{l}0,205 \\
4\end{array}$ & $\begin{array}{l}0,103 \\
4\end{array}$ & $\begin{array}{l}0,14 \\
4\end{array}$ & $\begin{array}{l}0,1 \\
4\end{array}$ \\
\hline \multirow[t]{3}{*}{ OR3 } & $\begin{array}{l}\text { Valeur } \\
\text { Classe }\end{array}$ & $\begin{array}{l}0,1 \\
4\end{array}$ & $\begin{array}{l}0,149 \\
4\end{array}$ & $\begin{array}{l}0,1 \\
4\end{array}$ & $\begin{array}{l}0,215 \\
4\end{array}$ & $\begin{array}{l}0,169 \\
4\end{array}$ & $\begin{array}{l}0,2 \\
4\end{array}$ & $\begin{array}{l}0,13 \\
4\end{array}$ \\
\hline & & Nitrites (valeurs en $\mu \mathrm{g} / \mathrm{L}$ ) & & & & & & \\
\hline & & Janvier & Février & Mars & Mai & Juin & Août & Octobre \\
\hline OR1 & $\begin{array}{l}\text { Valeur } \\
\text { Classe }\end{array}$ & $\begin{array}{l}0 \\
5\end{array}$ & $\begin{array}{l}5,63 \\
5\end{array}$ & 22,5 & $\begin{array}{l}16,9 \\
3\end{array}$ & 11,2 & 53,4 & 16,9 \\
\hline \multirow[t]{2}{*}{ OR2 } & Valeur & 0 & 0 & 16,9 & 33,7 & 0 & 42,2 & 19,7 \\
\hline & Classe & 5 & 5 & 3 & 3 & 5 & 3 & 3 \\
\hline \multirow[t]{3}{*}{ OR3 } & Valeur & 22 & 0 & 56,3 & 33 & 11,2 & 30,9 & 5,6 \\
\hline & Classe & $\begin{array}{l}3 \\
\text { Orthophosphates } \\
\text { (valeurs en } \mu \mathrm{g} / \mathrm{L} \text { ) }\end{array}$ & 5 & 2 & 3 & 3 & 3 & 5 \\
\hline & & Janvier & Février & Mars & Mai & Juin & Août & Octobre \\
\hline \multirow[t]{2}{*}{ OR1 } & Valeur & 0 & 18,4 & 2,6 & 10,5 & 1,9 & 34,9 & 40,9 \\
\hline & Classe & 5 & 4 & 5 & 5 & 5 & 4 & 4 \\
\hline \multirow[t]{2}{*}{ OR2 } & Valeur & 34,3 & 25 & 5,3 & 5,9 & 9,2 & 27,7 & 37,6 \\
\hline & Classe & 4 & 5 & 5 & 5 & 5 & 4 & 4 \\
\hline \multirow[t]{3}{*}{ OR3 } & Valeur & 0 & 15,7 & 4 & 1,32 & 5,3 & 3,9 & 56,1 \\
\hline & Classe & $\begin{array}{l}5 \\
\text { IPO }\end{array}$ & 5 & 5 & 5 & 5 & 5 & 4 \\
\hline & & Janvier & Février & Mars & Mai & Juin & Août & Octobre \\
\hline \multirow[t]{2}{*}{ OR1 } & Valeur & 4,25 & 3,75 & 4,25 & 4 & 4 & 3,5 & 4 \\
\hline & Classe & Faible & Modérée & Faible & Faible & Faible & Modérée & Faible \\
\hline \multirow[t]{2}{*}{ OR2 } & Valeur & 3,75 & 3,75 & 3,5 & 3,25 & 3,75 & & 3 \\
\hline & Classe & Modérée & Modérée & Modérée & Modérée & Modérée & Modérée & Modérée \\
\hline \multirow[t]{2}{*}{ OR3 } & Valeur & 3,25 & 3,5 & 3 & 3,25 & 3,25 & 3,25 & 3,5 \\
\hline & Classe & Modérée & Modérée & Modérée & Modérée & Modérée & Modérée & Modérée \\
\hline
\end{tabular}

s'éloigne plus loin en aval du barrage à O3, la richesse spécifique augmente pour revenir au niveau d'O1. La présence du barrage semble donc agir sur les peuplements de plécoptères en réduisant leur diversité. Deux taxa sont nouveaux $(H$. flaviventris et les Taeniopterygidae). Le stade juvénile des spécimens de Taeniopterygidae ne nous a pas permis de faire une identification plus poussée, c'est la première fois que cette famille est recensée dans les stations; les Taeniopterygidae sont présents ailleurs dans le Haut-Atlas avec deux espèces, Brachyptera algirica (Aubert, 1956) et Brachyptera 
Tableau 5. Indice de Pollution Organique (IPO) à l'Oukaïmeden.

Table Organic Pollution Index (IPO) in Oukaïmeden.

\begin{tabular}{|c|c|c|c|c|c|c|c|c|}
\hline & DBO5 (mg/L) & & & & & & & \\
\hline & Janvier & Février & Mars & Mai & Juin & Août & Octobre & Novembre \\
\hline O1 Valeur & 5,7 & 8 & 4 & 7,3 & 7,1 & 9 & 6 & 2 \\
\hline Classe & 3 & 3 & 4 & 3 & 3 & 3 & 3 & 4 \\
\hline O2 Valeur & 16,25 & 25,84 & 20,15 & 14,76 & 15,38 & 20 & 18,38 & 17 \\
\hline Classe & 1 & 1 & 1 & 2 & 1 & 1 & 1 & 1 \\
\hline O3 Valeur & 6,5 & 10,75 & 5 & 10 & 9 & 10 & 9,83 & 4 \\
\hline \multirow{3}{*}{ Classe } & 3 & 2 & 4 & 3 & 3 & 3 & 3 & 4 \\
\hline & $\begin{array}{l}\text { Ammonium } \\
\text { (mg/L) }\end{array}$ & & & & & & & \\
\hline & Janvier & Février & Mars & Mai & Juin & Août & Octobre & Novembre \\
\hline O1 Valeur & 0,02 & 0,1 & 0,05 & 0,08 & 0,05 & 0,02 & 0,035 & 0,02 \\
\hline Classe & 5 & 4 & 5 & 5 & 5 & 5 & 5 & 5 \\
\hline O2 Valeur & 0,04 & 0,234 & 0,07 & 0,03 & 0,04 & 0,09 & 0,051 & 0,05 \\
\hline Classe & 5 & 4 & 5 & 5 & 5 & 5 & 5 & 5 \\
\hline O3 Valeur & 0,02 & 0,091 & 0,04 & 0,06 & 0,04 & 0,05 & 0,035 & 0,03 \\
\hline \multirow{3}{*}{ Classe } & 5 & 5 & 5 & 5 & 5 & 5 & 5 & 5 \\
\hline & Nitrite $(\mu g / L)$ & & & & & & & \\
\hline & Janvier & Février & Mars & Mai & Juin & Août & Octobre & Novembre \\
\hline O1 Valeur & 0 & 28 & 5,63 & 16,9 & 22 & 19,7 & 20 & 30,9 \\
\hline Classe & 5 & 3 & 5 & 3 & 3 & 3 & 3 & 3 \\
\hline O2 Valeur & 12 & 188 & 0 & 50,6 & 73 & 56,3 & 20 & 16,9 \\
\hline Classe & 3 & 1 & 5 & 3 & 2 & 4 & 4 & 3 \\
\hline O3 Valeur & 0 & 90 & 0 & 28,1 & 10 & 2,8 & 0 & 64,7 \\
\hline \multirow[t]{3}{*}{ Classe } & 5 & 2 & 5 & 3 & 4 & 5 & 5 & 2 \\
\hline & $\begin{array}{l}\text { Orthophosphate } \\
(\mu \mathrm{g} / \mathrm{L})\end{array}$ & & & & & & & \\
\hline & Janvier & Février & Mars & Mai & Juin & Août & Octobre & Novembre \\
\hline O1 Valeur & 0 & 0,0039 & 0,0099 & 0,00792 & 0,0198 & 0 & 0,017 & 0,0283 \\
\hline Classe & 5 & 5 & 5 & 5 & 4 & 5 & 4 & 4 \\
\hline O2 Valeur & 14,5 & 0 & 89,7 & 2,64 & 14,5 & 45,5 & 47 & 64 \\
\hline Classe & 5 & 5 & 3 & 5 & 5 & 4 & 4 & 4 \\
\hline O3 Valeur & 10,5 & 42,9 & 0 & 16,5 & 23,1 & 62,7 & 0 & 1,3 \\
\hline Classe & $\begin{array}{l}5 \\
\text { IPO }\end{array}$ & 4 & 5 & 4 & 3 & 4 & 5 & 5 \\
\hline & Janvier & Février & Mars & Mai & Juin & Août & Octobre & Novembre \\
\hline O1 Valeur & 4,5 & 3,75 & 4,75 & 4 & 3,75 & 4 & 3,75 & 4 \\
\hline Classe & Faible & Modérée & Nulle & Faible & Modérée & Faible & Modérée & Faible \\
\hline $\mathrm{O} 2$ Valeur & 3,75 & 2,75 & 3,5 & 3,75 & 3,25 & 3,5 & 3,5 & 3,25 \\
\hline Classe & Modérée & Forte & Modérée & Modérée & Modérée & Modérée & Modérée & Modérée \\
\hline O3 Valeur & 4,5 & 3,25 & 4,75 & 3,75 & 3,75 & 4,25 & 4,5 & \\
\hline Classe & Faible & Modérée & Nulle & Modérée & Modérée & Faible & Faible & Faible \\
\hline
\end{tabular}

auberti (Consiglio, 1957), il est probable que les individus récoltés appartiennent à l'une de ces espèces.

À l'instar de Nigrobaetis, Hemimelaena flaviventris a la particularité d'avoir colonisé les stations des deux cours d'eau après 2000, alors qu'elle était absente avant 2000. L'espèce en expansion dans la zone d'étude semble également en expansion au Maroc où sa présence a été nouvellement recensée dans plusieurs cours 


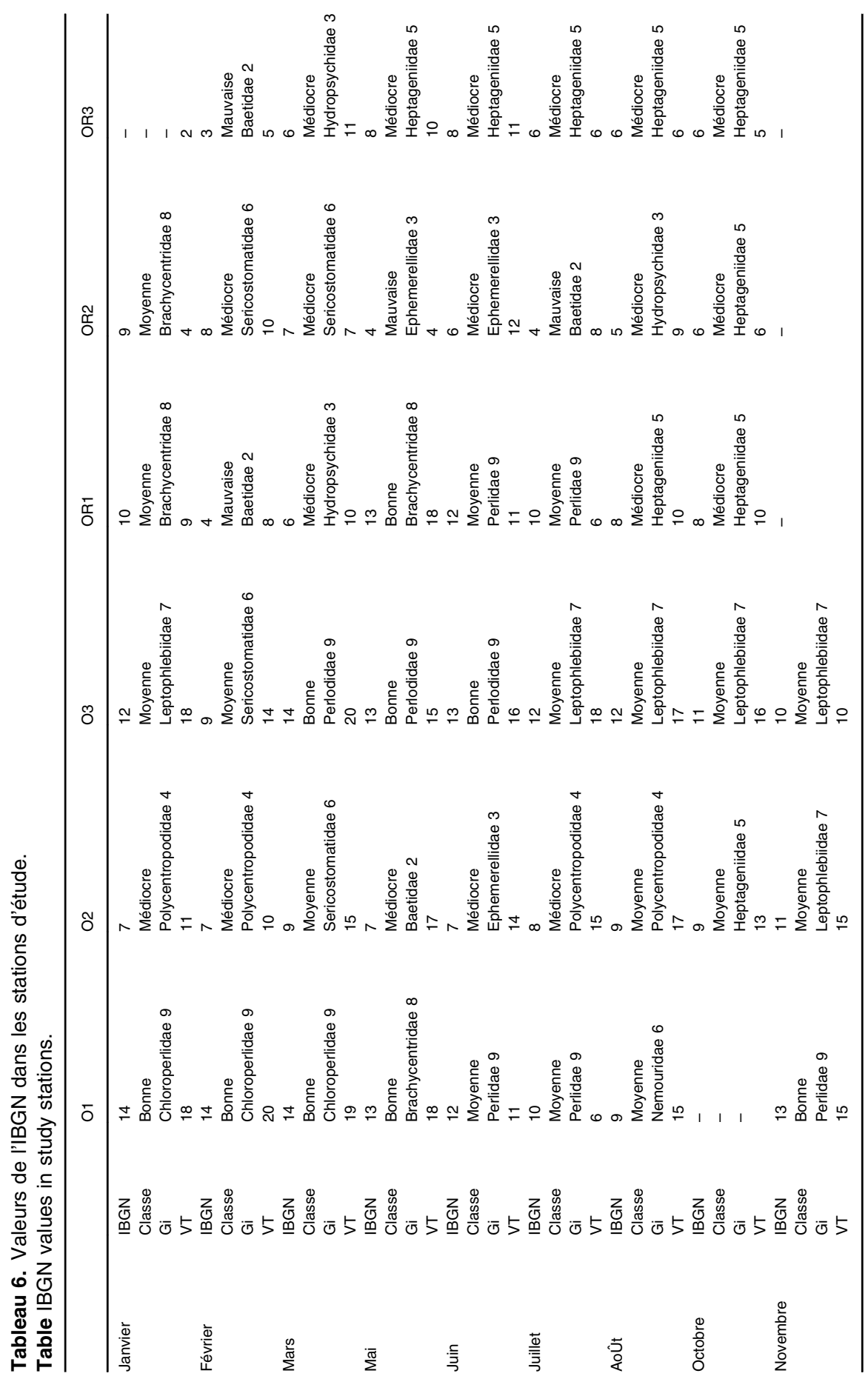


d'eau au Nord-Ouest du pays (El Bazi et al., 2017). H. flaviventris est une espèce endémique de la péninsule Ibérique et du Maroc (Dakki 1987; Sánchez-Ortega et al., 2003; Errochdi et al., 2014).

De même que pour les éphéméroptères, nous constatons donc que la diversité des plécoptères est désormais plus importante dans à haute altitude (Oukaïmeden), tandis qu'elle a baissé à moyenne altitude (Ourika).

\subsubsection{Trichoptères}

Au total, 19 espèces de trichoptères ont été recensées dans les stations prospectées (Tab. 5). Cela représente $26 \%$ des trichoptères du Maroc (73 espèces), avec notamment Agapetus dolichopterus endémique du Maroc et Hydropsyche fezana endémique du Maroc et de l'Algérie.

À l'Ourika, la baisse de la richesse spécifique est très importante. Près de la moitié des espèces recensées avant 2000 ont disparu des stations, passant de 17 espèces avant 2000, à 10 après 2000. Cheumatopsyche atlantis, Plectronemia laetabilis, Stenophylax espanioli, Hydroptila vectis, Orthotrichia angustella, Psychomyia pusilla et la famille des Phryganidae ont disparu des stations, aucun nouveau taxon n'a été recensé après 2000 .

À l'Oukaïmeden, 11 espèces étaient recensées avant 2000 , contre 10 après 2000. Deux espèces sont nouvelles au sein des stations (H.lobata et $H$. maroccana), 3 ont disparu (P. laetabilis, S. espanioli et $H$.vectis). La présence du barrage ne semble pas avoir d'effet particulier sur les peuplements de trichoptères, la station en amont
(O1) et les stations en aval (O2 et O3) ayant une richesse spécifique à peu près identique.

À l'Oukaïmeden, la richesse spécifique des trichoptères est restée à peu près identique, tandis qu'à l'Ourika, elle a considérablement diminué.

\subsection{L'habitat aquatique, influence sur les peuplements}

Parmi les paramètres qui influent sur la diversité spécifique des peuplements étudiés, l'habitat aquatique occupe une place majeure, la diversité biologique des rivières est étroitement liée à la qualité et la quantité de l'habitat physique disponible. Dans un écosystème non perturbé, les caractéristiques d'habitat constituent les principaux facteurs qui déterminent la distribution des macros invertébrés (Hynes, 1970; Cummins, 1975; Hellawell, 1986). Les résultats de l'évaluation de l'habitat aquatique par l'Indice de qualité de l'habitat (IQH) sont présentés dans les tableaux 2 et 3.

Dans la station OR1, l'habitat aquatique est globalement marginal avec toutefois une amélioration nette en janvier et en mai où il est sous-optimal. L'insuffisance à pouvoir accueillir et maintenir des peuplements diversifiés résulte principalement des modifications que subit le cours d'eau. Ce dernier est canalisé et dragué pour divers aménagements anthropiques, ce qui rend le substrat benthique instable. La végétation rivulaire est aussi désormais quasi absente, contrairement à la période avant 2000 où elle était constituée d'une ripisylve herbacée et boisée (peupliers, noyers). 
Dans la station OR2, l'habitat aquatique est globalement sous-optimal. Cependant, bien que la station dispose d'un substrat benthique adéquat pour accueillir et maintenir les peuplements, elle subit des dragages fréquents qui entraînent une disparition mécanique des taxa. L'envasement augmente en été et la qualité de l'habitat connait une nette régression en juillet.

Dans la station OR3, l'habitat aquatique est marginal. Bien que le substrat benthique soit diversifié, l'ensablement et l'envasement sont particulièrement importants. Le cours d'eau subit également des modifications avec une canalisation, des remblais et un dragage important. De plus, il n'y a pas de protection végétale des berges.

Dans la station 01, l'habitat aquatique est sous-optimal. La faible largeur du cours d'eau limite le nombre de couples vélocité-profondeur, les régimes lent-profond et rapide-profond sont absents, ce qui limite la présence des espèces ayant une préférence pour ces régimes. En hiver et en automne, le degré de marnage est faible tandis qu'au printemps une partie de l'eau de la station est déviée mais le marnage reste élevé. La régulation du pâturage avec la mise en défens entre mai et juillet permet à la végétation rivulaire de se développer renforçant ainsi la protection végétale des berges.

Dans la station O2, I'habitat est sousoptimal, mais avec un substrat benthique moins diversifié par rapport à $\mathrm{O} 1$ et $\mathrm{O} 3$ où l'hétérogénéité est plus marquée entre blocs, galets et graviers. La présence du barrage semble limiter la diversité de substrat benthique. Les seuils sont également moins nombreux limitant l'oxygénation du milieu. La station connaît un envasement important entre le printemps et l'automne. L'instabilité de la rive gauche contribue à cet apport de sédiments fins ainsi que de gravats, qui constituent en parti le substrat benthique avec un mélange d'habitats stables et d'habitats insuffisants. La végétation rivulaire est plus importante entre juin et juillet.

Dans la station O3, I'habitat est globalement sous-optimal. II est limité par la présence de fosses, des couples vélocité-profondeur peu variés, et une faible protection végétale des berges qui s'améliore toutefois entre mai et juillet.

Les rives des stations de l'Ourika ont beaucoup évolué au fil des années. Avant 2000, elles étaient beaucoup plus végétalisées, avec essentiellement une végétation herbacée et des cultures, notamment à la station OR1 qui présentait des cultures arborées de peupliers et de noyers. Cette végétation rivulaire remplissait de nombreuses fonctions écologiques favorisant la vie aquatique (rétention des sédiments, stabilisation des berges, apport d'éléments nutritifs...), en plus de préserver la faune aquatique lors des crues. Les événements de crues de l'ordre de $103 \mathrm{~m}^{3} / \mathrm{s}$, se produisent en moyenne tous les deux ans et des débits de pointe de l'ordre de $485 \mathrm{~m}^{3} / \mathrm{s}$ reviennent tous les 10 ans (Saidi El Mehdi et al., 2003). Mais ce sont d'abord les crues de faible intensité, plus fréquentes, qui impactent de manière récurrente la faune. Les crues à l'Ourika résultent généralement de fortes averses localisées dans l'espace. Les observations faites sur une longue série d'épisodes de crues 


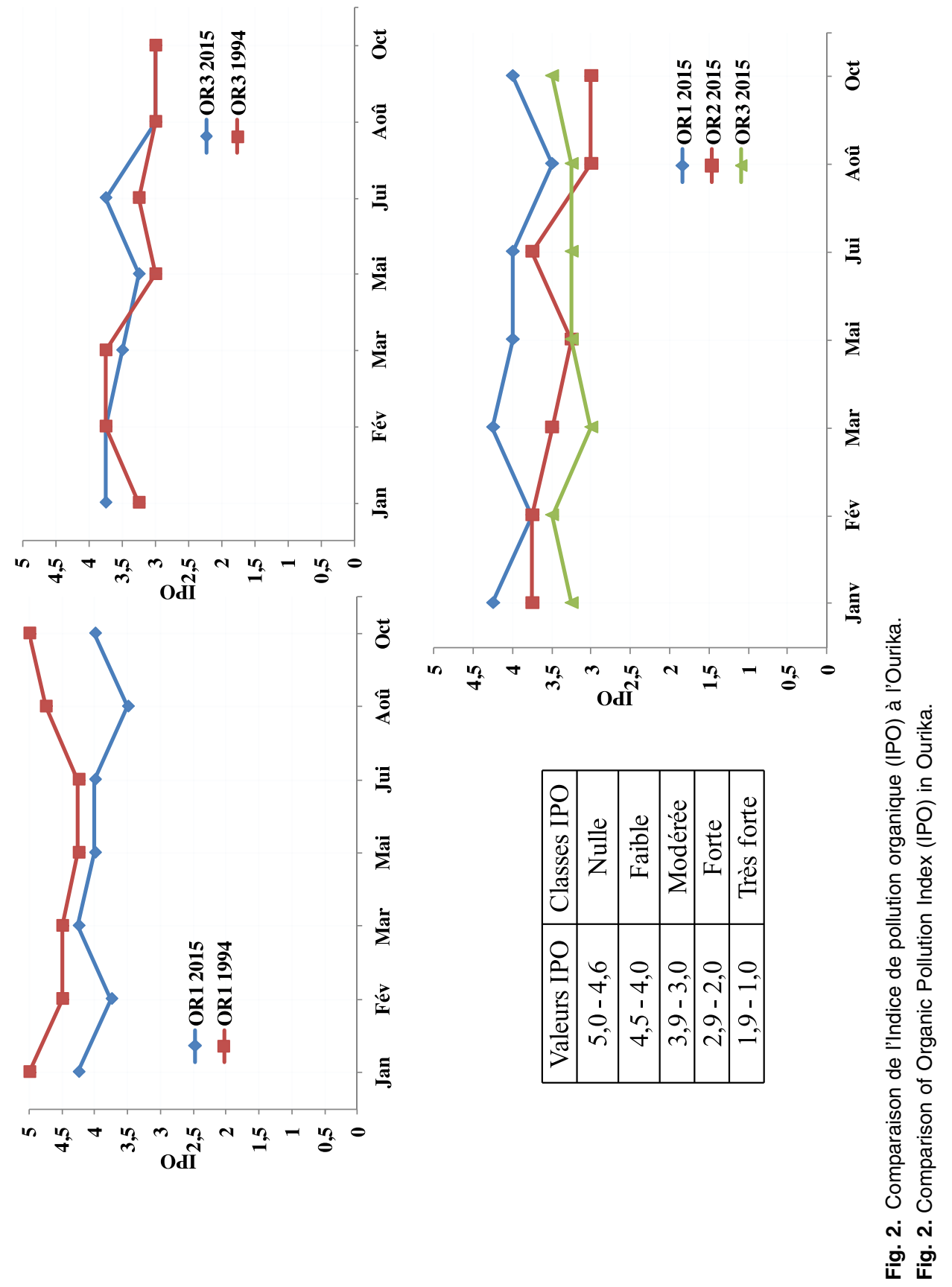



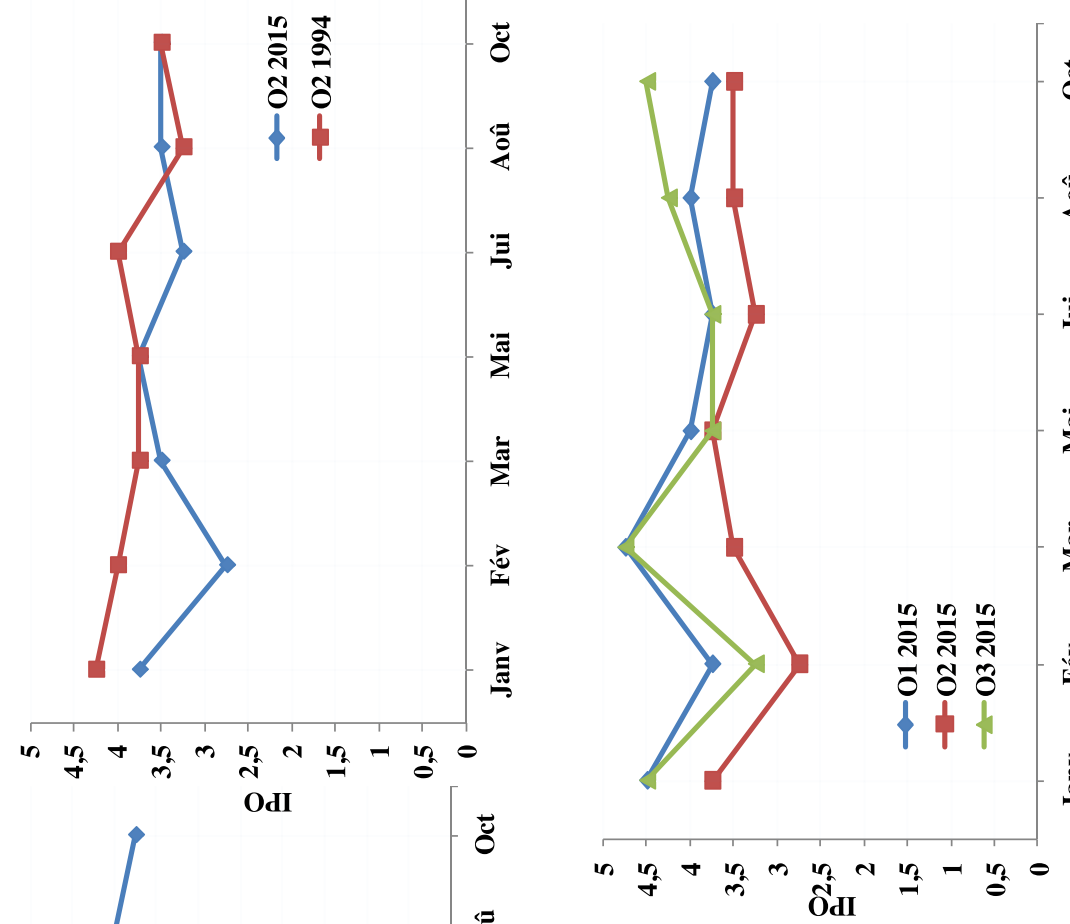

\section{$\bar{\Xi}$}

9

$\Xi$

茫

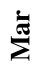

屋

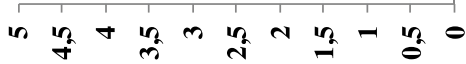

OdI

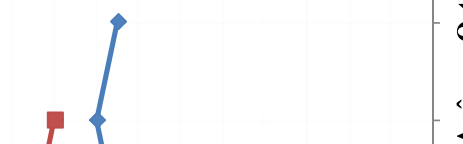

$\stackrel{\vec{E}}{\rightarrow}$

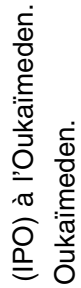

$\Xi$

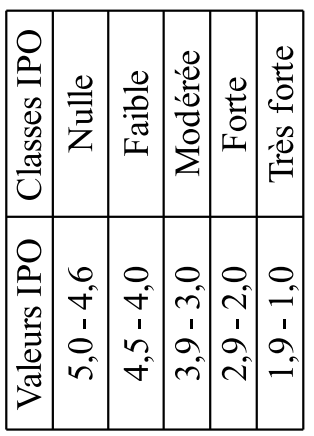

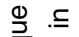

흔

"ే

齐

蛋

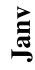

잉

등 임

흥 윽

응 흥

¿

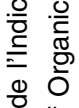

응

당

용

뜬

है है

लं

은 훈 
montrent que celles-ci surviennent en toutes saisons et que chaque mois (hormis décembre) a déjà connu au moins une crue (Saidi El Mehdi et al., 2010). Au cours de l'année le printemps est la période où les crues sont les plus fréquentes. L'été pourtant période de sécheresse mérite également d'être signalé comme une saison à risque (Saidi El Mehdi et al., 2010). Les stations de l'Oukaïmeden en revanche ne sont pas soumises à des épisodes de crues intenses comme celles observées à l'Ourika, la végétation rivulaire a également peu évolué.

\subsection{La pollution organique}

Les données chimiques variant rapidement au cours du temps, les résultats des analyses chimiques ne témoignent que de la composition de l'eau au moment de l'échantillonnage (Resh et al., 1996). II n'existe que très peu de données sur la pollution organique dans la zone d'étude. Les travaux menés par (Talami 1998) ont, néanmoins, permis de renseigner le niveau de pollution organique antérieur à 2000. Les tableaux 4 et 5 présentent les résultats de l'évaluation de la pollution par I'IPO à l'Ourika et à l'Oukaïmeden.

\subsubsection{Ourika}

À l'Ourika, dans la station OR1, la pollution organique varie entre faible et modérée (Tab. 4). Les mesures effectuées par Talami (1998) avant 2000 montrent qu'elle variait entre les classes nulle et faible (Fig. 2), la pollution organique a donc augmenté.
Dans la station OR2, le niveau de pollution antérieur à 2000 n'est pas connu. Les mesures effectuées au cours de cette étude révèlent qu'elle est modérée tout au long de l'année. La station étant située en aval de la confluence avec l'Oued Oukaïmeden, la pollution organique est donc étroitement liée aux apports dans la partie terminale ce dernier.

Dans la station OR3, la pollution organique est modérée tout au long de l'année. Avant 2000, elle variait entre forte et modéré, elle a gardé des proportions à peu près similaires.

\subsubsection{Oukaïmeden}

À l'Oukaïmeden, dans la station 01 , la pollution organique varie entre les classes nulle et modérée (Tab. 5). Les évaluations antérieures à 2000 (Talami 1998) ont montré qu'elle variait entre les classes nulle et faible (Fig. 3), elle a donc augmenté. Malgré cette augmentation, le niveau de pollution au sein de la station reste suffisamment bas pour permettre à une faune riche et variée de se développer.

Dans la station $\mathrm{O} 2$, la pollution organique varie entre les classes forte et modérée. Les mesures effectuées avant 2000 (Talami, 1998) montrent qu'elle variait entre les classes faible et modérée (Fig. 3). II y a donc eu une augmentation de la pollution organique, ce qui a contribué à rendre le milieu aquatique moins favorable à la macrofaune benthique. Le barrage ne semble pas avoir une influence majeure sur le niveau de pollution organique.

Dans la station 03, le niveau de pollution organique antérieure à 2000 n'est pas connu. L'évaluation effectuée 

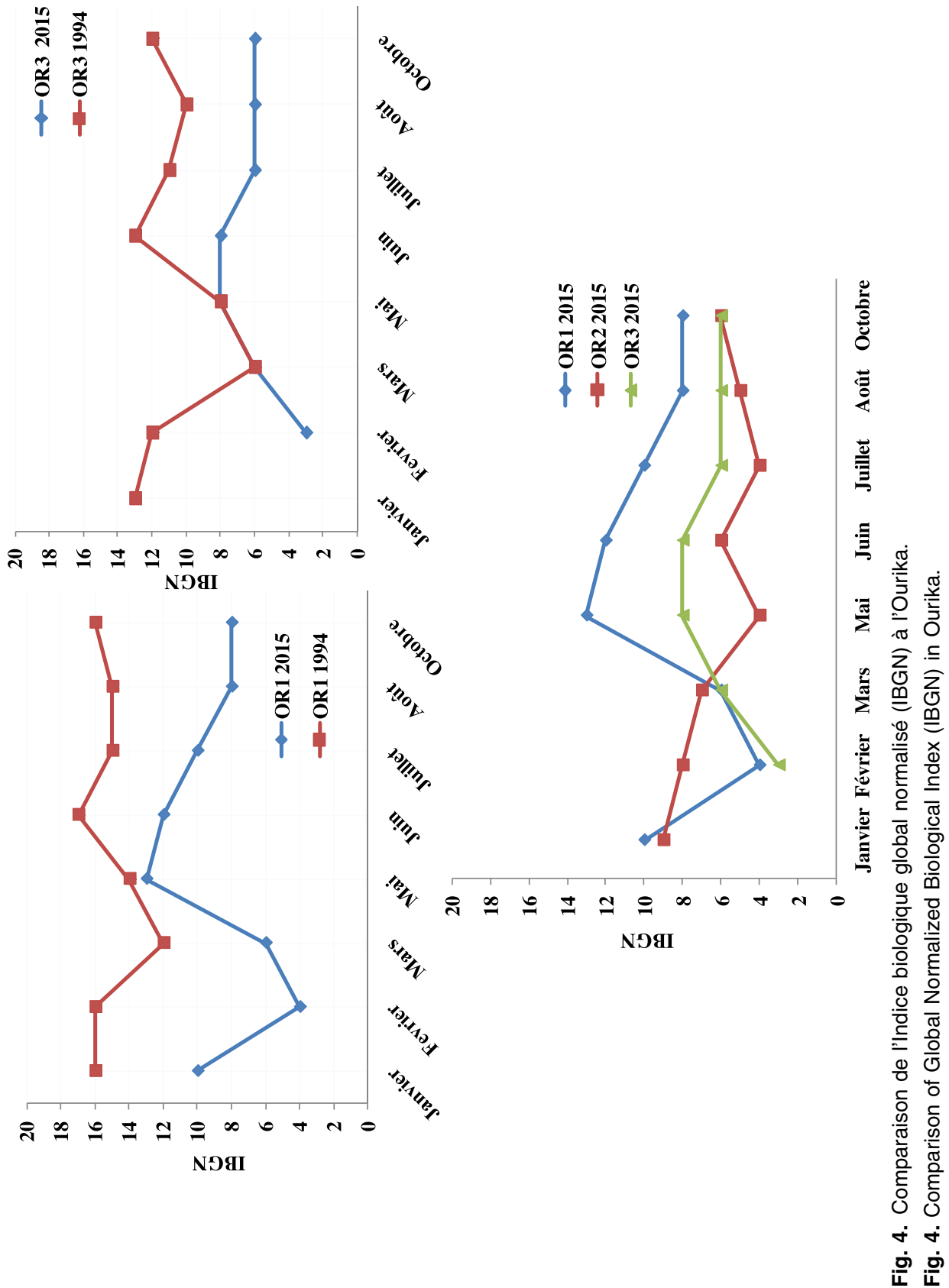


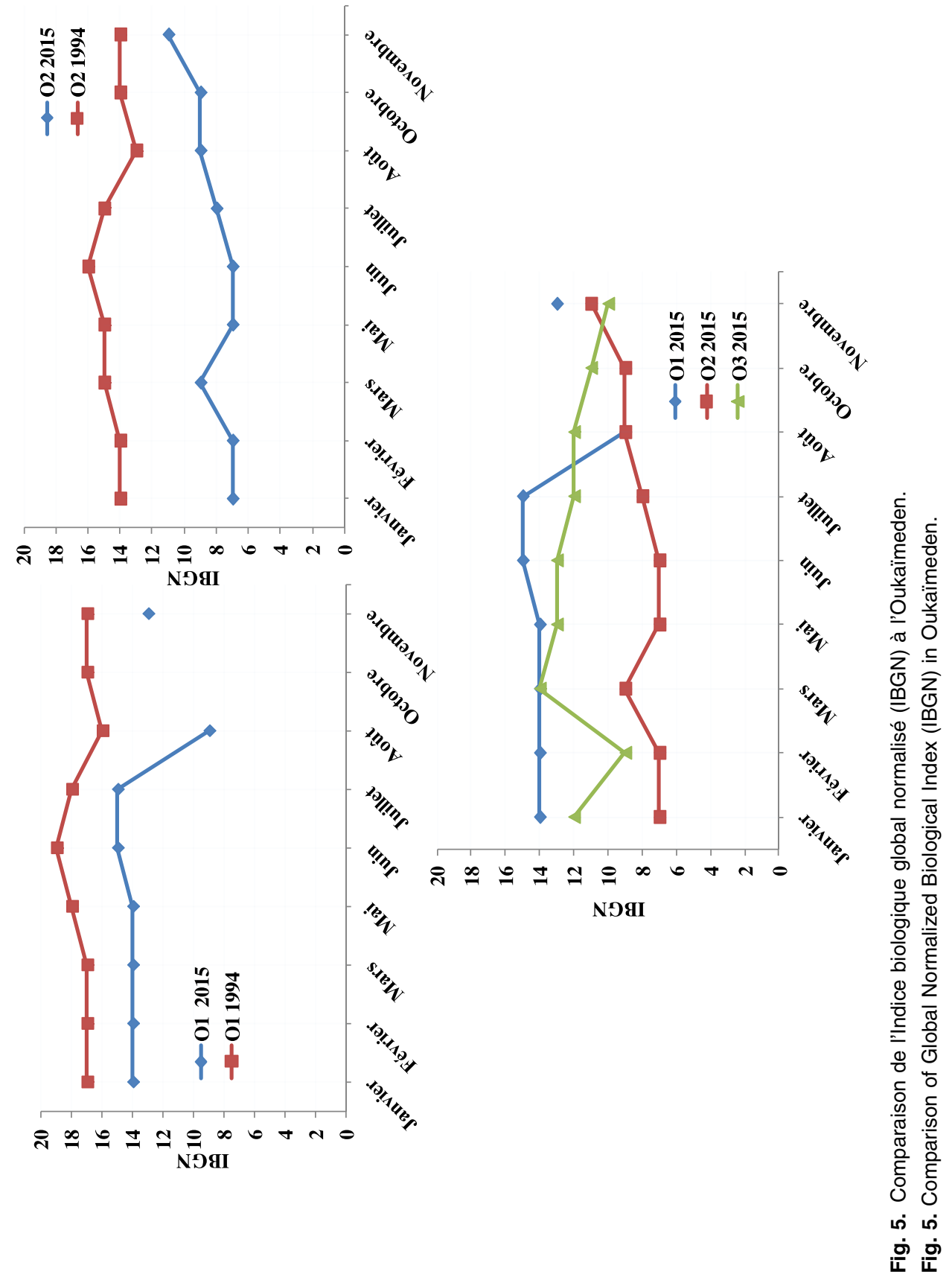


révèle qu'elle varie entre les classes modérée et nulle. La macrofaune benthique est donc relativement peu impactée. La station étant en aval de la confluence avec l'assif Tiferguine, la pollution organique dépend des apports de la partie terminale de ce dernier.

\subsection{La qualité biologique de l'eau}

La qualité de l'eau influe sur la richesse taxonomique (Parsons \& Norris 1996; Iliopoulou-Georgudaki et al., 2003; Soldner et al., 2004), et les macroinvertébrés benthiques sont de bons indicateurs de la pollution de l'eau (Hilsenhoff 1988; Lenat 1988; Plafkin et al., 1989; Klemm et al., 1990; Bode et al., 1991; Cayrou et al., 2000). II existe très peu de données sur la qualité de l'eau antérieures à cette étude. Les évaluations de la qualité biologique de l'eau menée par Talami (1998) ont servi de point de comparaison pour déterminer l'évolution de ce paramètre.

\subsubsection{Ourika}

Les résultats de l'évaluation de la qualité biologique de l'eau par l'IBGN sont présentés dans le tableau 6 .

Dans la station OR1, la qualité biologique de l'eau est moins dégradée entre mai et juillet, avec une bonne qualité en mai. Le reste de l'année, l'eau est de qualité biologique médiocre avec une régression en février (mauvaise qualité). C'est donc de mai à juillet que la qualité biologique de l'eau semble favorable aux peuplements benthiques dans la station OR1.

Dans la station OR2, l'eau est souvent de qualité biologique médiocre, avec une régression en mai et en juillet où elle est de mauvaise qualité.
II y a une amélioration en janvier où l'eau est de qualité biologique moyenne. Les faibles valeurs de l'IBGN enregistrées tout au long de l'année dans la station OR2 mettent en évidence un milieu aquatique peu favorable à des peuplements benthiques riches et diversifiés.

Dans la station OR3, la qualité biologique de l'eau est souvent médiocre tout au long de l'année, avec une régression entre janvier et février (hors classes en janvier et mauvaise en février). La très faible présence de macroinvertébrés benthiques en janvier ne permet pas le calcul de l'IBGN. $\mathrm{Au}$ niveau de cette station, le milieu aquatique est particulièrement défavorable aux peuplements benthiques.

Les résultats de I'IBGN montrent donc que la qualité biologique de l'eau est très dégradée à l'Ourika, particulièrement en aval d'OR1.

La comparaison avec les évaluations effectuées avant 2000 (Talami, 1998) montre qu'au niveau de la station OR1, il y a eu une dégradation considérable de la qualité biologique de l'eau. Nous sommes passés d'une qualité biologique variant entre bonne et excellente, à une qualité biologique variant désormais entre bonne et médiocre (Fig. 4).

Dans la station OR2, les valeurs de I'IBGN antérieures à 2000 ne sont pas connues. Dans la station OR3, la qualité biologique de l'eau a également connu une régression particulièrement importante, elle variait entre bonne et moyenne, elle varie désormais entre mauvaise et médiocre.

\subsubsection{Oukaïmeden}

Les stations de l'Oukaïmeden ont un IBGN plus élevé que celui de l'Ourika, 
ce qui traduit une meilleure qualité biologique de l'eau dans cette région.

Dans la station 01, la qualité biologique de l'eau est bonne, sauf entre juin et août où elle régresse et est moyenne. Le milieu aquatique est globalement favorable au développement d'une macrofaune benthique riche et variée.

Dans la station $\mathrm{O} 2$, de janvier à juin, la qualité biologique de l'eau est globalement médiocre, de juillet à novembre il y a une nette amélioration, et la qualité biologique est moyenne. Le milieu aquatique est beaucoup moins favorable à une macrofaune benthique diversifiée, en particulier pour les espèces sensibles à la pollution.

Dans la station O3, la qualité biologique de l'eau est moyenne de janvier à février, puis il y a une amélioration et la qualité biologique est bonne de mars à juin. II y a ensuite une régression et la qualité biologique est à nouveau moyenne de juillet à novembre. Le milieu aquatique est donc plus favorable à des peuplements benthiques riches et diversifiés durant la période de printemps. Le reste de l'année le milieu aquatique est moins favorable aux espèces les plus polluosensibles.

La comparaison avec les évaluations antérieures à 2000 (Fig. 5) montre qu'à l'Oukaïmeden, dans la station O1, il y a eu une régression de la qualité biologique de l'eau. Talami (1998) relevait qu'elle oscillait entre bonne et excellente, elle varie désormais entre moyenne et bonne. Malgré cette régression, la qualité biologique de l'eau demeure favorable et des espèces les plus polluosensibles restent présentes.
Dans la station O2, Talami (1998) relevait que la qualité biologique de l'eau était bonne tout au long de l'année, elle oscille désormais entre moyenne et médiocre. II y a donc eu une forte régression de la qualité biologique de l'eau au sein de cette station, le milieu est désormais peu favorable à la colonisation par une macrofaune benthique diversifiée, en particulier pour les espèces sensibles à la pollution. La bonne qualité biologique de l'eau relevée avant 2000 montre que l'impact du barrage sur la communauté est relativement faible. Cependant, la présence du barrage semble impacté fortement les espèces les plus sensibles aux perturbations, ce qui se traduit notamment par la très faible présence des plécoptères à $\mathrm{O} 2$, alors qu'à $\mathrm{O} 1$ en amont, et à $\mathrm{O} 3$ plus loin en aval, leur présence est beaucoup plus importante. À O3 la qualité de l'eau pour la période antérieure à 2000 n'est pas connue.

\section{Discussion}

Éphéméroptères, plécoptères et Trichoptères (EPT) sont généralement considérés comme les groupes les plus sensibles à la pollution et aux perturbations (Klemm et al., 1990; Resh et al., 1995; Barbour et al., 1999). Un nombre peu élevé de taxa EPT est généralement liée à une augmentation des perturbations. À l'inverse, un nombre élevé de taxa EPT est généralement le signe d'un cours d'eau en bonne santé.

L'analyse de la richesse spécifique a montré qu'avant 2000, il y a avait plus d'espèces EPT dans les stations de 

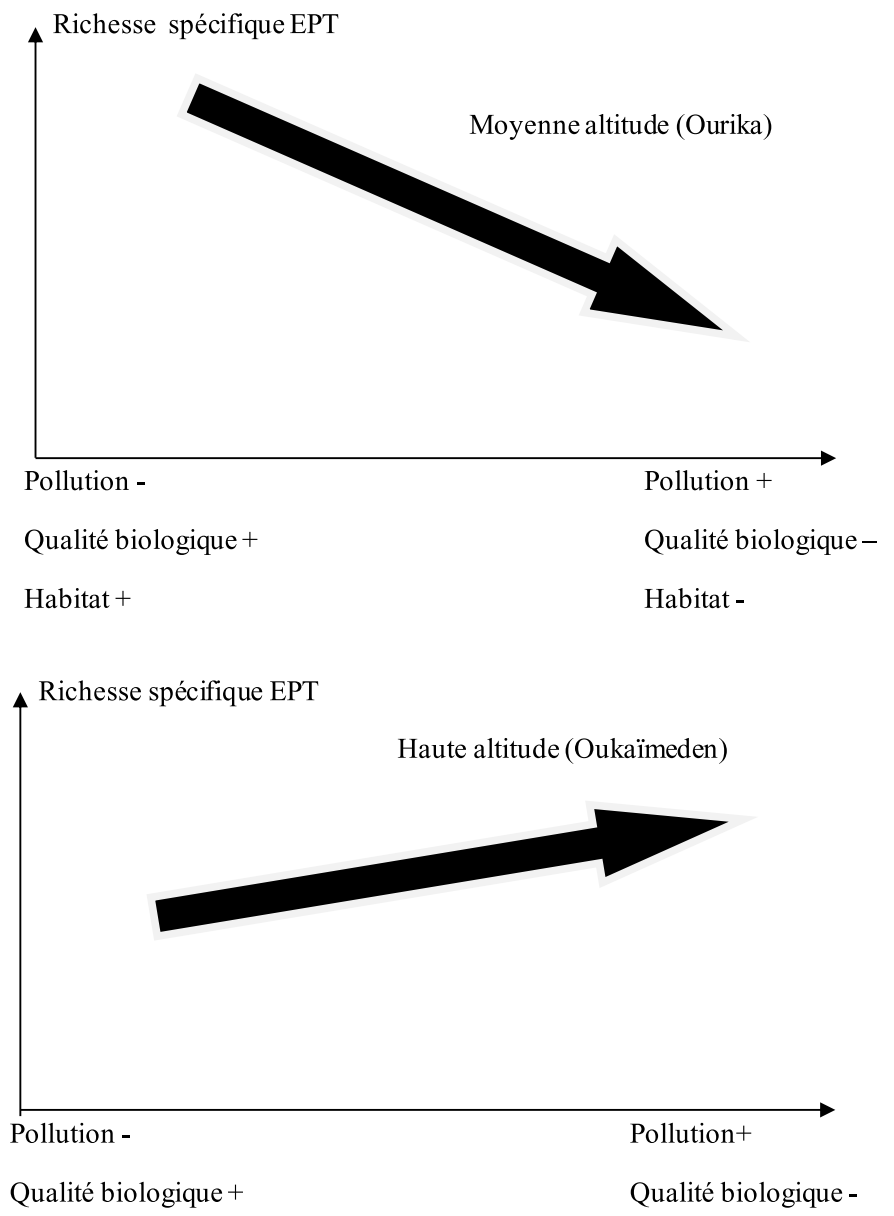

Fig. 6. Évolution des composantes de la qualité globale du milieu aquatique et impact sur la richesse spécifique (EPT).

Fig. 6. Evolution of the components of the overall quality of the aquatic environment and impact on the specific richness (EPT).

l'Ourika qui sont à des altitudes moyennes, que dans celles de l'Oukaïmeden qui sont à des altitudes beaucoup plus élevées. Trente-sept espèces EPT étaient en effet recensées à l'Ourika, contre 27 à l'Oukaïmeden. Il y avait une diminution du nombre d'espèces EPT avec l'altitude. La situation s'est inver- sée, le nombre d'espèces EPT est désormais plus important à l'Oukaïmeden. À l'Ourika, la richesse spécifique est passée de 37 espèces avant 2000 à 24 espèces après 2000 , soit une baisse de $35 \%$. À l'Oukaïmeden, la richesse spécifique est passée de 27 espèces avant 2000 à 32 après 2000 , soit une 
augmentation de $15 \%$. Il y a désormais une augmentation de la richesse spécifique avec l'altitude.

De façon générale, l'évolution de la qualité globale du milieu aquatique détermine l'évolution de la richesse spécifique. La qualité globale du milieu aquatique dépend de la qualité physique, chimique et biologique du milieu. La qualité chimique a été évaluée à travers la pollution organique (IPO), la qualité physique à travers l'habitat aquatique (IQH) et la qualité biologique à travers I'IBGN. La qualité chimique et la qualité physique du milieu impactent directement la qualité biologique. Nous pouvons également voir sur la figure 6 l'évolution des composantes après 2000 et les conséquences qui en ont découlé sur les peuplements benthiques à l'Ourika et à l'Oukaïmeden.

L'évaluation de l'habitat aquatique par l'IQH révèle que dans les stations de l'Ourika, l'habitat aquatique est marginal, excepté à OR2 où il est sous-optimal. À l'Ourika, l'habitat est souvent dégradé avec des perturbations anthropiques récurrentes. Cela contribue à expliquer la faible richesse spécifique relevé dans les stations. De plus, l'Ourika connaît souvent d'importantes crues qui ont des conséquences importantes sur les peuplements benthiques. Les crues ont effet des répercussions sur la structure des communautés (Townsend et al., 1997). En plus d'altérer l'habitat aquatique, les crues sont directement responsables de la diminution du nombre d'espèces, avec une modification de la densité et la distribution des populations (Croyle, 1997). L'impact des crues à l'Ourika n'est pas nouveau, mais autrefois, les stations étaient pourvues d'une bonne protection végétale des berges, ce qui jouait un rôle fondamental dans la recolonisation du milieu par la faune après la crue. Badri (1993) a menée des travaux relatifs à l'impact des crues sur les macroinvertébrés benthiques à l'Ourika. II a mis en évidence le fait que l'impact des crues sur la faune était atténué grâce à la végétation rivulaire, les taxa y étaient retenus durant la crue, c'était une zone refuge pour la faune. C'est à partir de cette faune refugiée dans la végétation rivulaire, et de la dérive, que le milieu aquatique était recolonisé par les macroinvertébrés benthiques. La végétation rivulaire étant désormais quasi absente à l'Ourika, cela a contribué à limiter la capacité de résilience du milieu, à réduire la capacité de recolonisation du milieu par la faune, et rendu plus vulnérable les peuplements benthiques.

À l'Oukaïmeden, l'habitat aquatique est sous-optimal, les perturbations sont beaucoup moins importantes. La régulation du pâturage avec la mise en défens entre mai et juillet permet à la végétation rivulaire de se développer, ce qui contribue à renforcer la capacité des bandes riveraines à remplir leurs fonctions écologiques en regard de la protection du milieu aquatique. Ainsi, l'habitat aquatique relativement peu dégradé à l'Oukaïmeden contribue à expliquer le fait que la richesse spécifique ait été conservée et y soit plus élevée.

Outre l'habitat aquatique, la chimie des eaux contribue également à expliquer la présence ou l'absence de certaines espèces animales et conditionne leur développement (Tuffery, 1980). L'Oukaïmeden est une région 
très peu anthropisée. L'impact de la pollution chimique avec notamment les rejets d'eaux usées domestiques est surtout concentré à la station $\mathrm{O} 2$ durant la période hivernale. La station $\mathrm{O} 2$ est située en aval d'un lac de barrage, or, des eaux usées domestiques sont rejetées au niveau de ce lac, particulièrement en hiver avec l'afflux touristique lié à la station de ski. L'impact de la pollution organique est donc plus important à $\mathrm{O} 2$, ce qui contribue à expliquer le fait que la diversité faunistique y soit plus faible par rapport aux autres stations de l'Oukaïmeden.

L'Ourika est beaucoup plus anthropisée, avec de nombreux restaurants juxtaposés au cours d'eau. La pollution organique y est plus importante, malgré un débit assez élevé qui a un certain effet de dilution sur les différents éléments chimiques contenus dans l'eau. La pollution organique est plus faible à OR1 par rapport aux autres stations de l'Ourika, ce qui contribue à une plus grande diversité faunistique au sein de cette station. Avant 2000, Mohati (1985) indiquait que la qualité de l'eau à l'Ourika était de plus en plus compromise et que des mesures devraient être prises pour la préserver. II évoquait le problème des eaux usées résultant des villages qui s'agrandissaient, et indiquait qu'il fallait éviter que ces eaux contaminent la rivière. II semble que les efforts de préservation du milieu aquatique de la pollution organique n'ont pas été suffisants et la pollution organique a augmenté.

L'impact de la pollution organique et de la qualité de l'habitat s'est répercuté sur la qualité biologique de l'eau. L'évaluation par I'IBGN a révélé que la qualité biologique de l'eau a connu une régression particulièrement importante par rapport à la période avant 2000, dans les stations de l'Ourika. L'absence de mesures adéquates pour limiter la pollution a grandement contribué à la régression de la qualité biologique de l'eau, ce qui se traduit par notamment une forte diminution de la richesse spécifique constatée au sein de ces stations.

L'Oukaïmeden a également connu une baisse de la qualité biologique de l'eau mais dans des proportions beaucoup moins importantes qu'à l'Ourika. Le milieu aquatique demeure favorable aux peuplements de macroinvertébrés benthiques excepté à la station $\mathrm{O} 2$.

La richesse spécifique des peuplements benthiques des rivières est étroitement liée à l'habitat aquatique, la qualité et la quantité d'habitat physique disponible ont une influence directe sur les peuplements. À l'Ourika, la dégradation de l'habitat aquatique et l'augmentation de la pollution contribue grandement à expliquer la disparition des espèces constatée. Cependant, d'autres facteurs ont pu intervenir et agir en synergie, contribuant à la baisse de la richesse spécifique constatée. Parmi ces facteurs, il y a en particulier le changement climatique. Les projections climatiques réalisées au sein du bassin de l'Ourika montrent en effet une augmentation progressive de la température de l'air depuis les années 1980 ainsi qu'une baisse des précipitations, ces deux tendances vont s'accentuer dans les prochaines années (Ayt Ougougdal et al., 2020). Avec le réchauffement climatique, on s'attend à une migration altitudinale des espèces entrainant une baisse de 
la richesse spécifique à moyenne altitude. Cependant, les changements climatiques actuels ont la particularité de se dérouler dans un contexte de destruction et de fragmentation des habitats sous l'impact anthropique, ce qui a tendance à masquer un éventuel impact du changement climatique sur les communautés. L'impact imputable au changement climatique sur le milieu aquatique étant souvent minoritaire par rapport à l'impact anthropique (Dunn et al., 2012; Floury et al., 2012).

À l'Oukaïmeden, la pollution organique est en augmentation et la qualité biologique de l'eau a régressé. De ce fait, bien que l'habitat aquatique ait peu varié, il semble que de façon globale, le milieu aquatique soit en légère régression. On devrait donc s'attendre à une baisse de la richesse spécifique à l'instar de ce qui est observé à l'Ourika, même si c'est dans des proportions moindres. Pourtant, le constat est que la richesse spécifique a augmenté. II y a donc probablement d'autres facteurs qui entrent en compte pour expliquer cette augmentation. Bien que cela n'ait pas été quantifié nous avons constaté à l'Oukaïmeden, à la différence de l'Ourika, une présence importante d'algues sur le substrat benthique, ainsi que de renoncules aquatiques. Une première hypothèse est que l'augmentation du nombre d'espèces serait liée à une présence d'algues et de végétation aquatique plus importante dans les stations de l'Oukaïmeden, par rapport à la période avant 2000 , en raison d'une augmentation des apports en matière organique. Cela aurait amélioré la disponibilité en ressources trophiques, contribuant ainsi à rendre les stations de l'Oukaïmeden plus attractives pour la faune. Une autre hypothèse est l'impact du changement climatique. La conséquence directe majeure du réchauffement de l'air est une augmentation de la température de l'eau pour les cours d'eau (Mohseni \& Stefan, 1999). L'une des réponses attendues de la faune face au réchauffement climatique est une migration altitudinale des espèces, on s'attend donc à une augmentation de la richesse spécifique dans les milieux haute altitude. Si l'impact éventuel du changement climatique sur la richesse spécifique à l'Ourika est masqué par les pressions anthropiques, il devrait être plus visible à l'Oukaïmeden. Ainsi, l'augmentation de la richesse spécifique observée en dépit d'une légère régression de la qualité du milieu aquatique pourrait résulter d'une migration altitudinale des espèces sous l'impact du réchauffement climatique.

\section{Conclusion}

L'analyse de l'évolution de la richesse spécifique des éphéméroptères, plécoptères et trichoptères révèle essentiellement deux tendances, d'une part, une diminution globale de la diversité dans les stations de l'Ourika qui sont situées à moyenne altitude, d'autre part, une augmentation de la richesse spécifique dans les stations de l'Oukaïmeden situées à des altitudes beaucoup plus élevées. La richesse spécifique des peuplements étudiés était plus importante à l'Ourika avant 2000. La situation s'est inversée et la richesse spécifique est désormais plus importante à l'Oukaïmeden. Cette 
situation s'explique en grande partie par le fait que le milieu aquatique s'est considérablement dégradé à l'Ourika sous la pression anthropique. L'habitat aquatique est de plus en plus altéré, la qualité biologique de l'eau a connu une régression considérable par rapport à la période avant 2000 et la pollution organique est assez importante. À l'Oukaïmeden en revanche, hormis à la station $\mathrm{O} 2$, le milieu aquatique et beaucoup moins perturbé, l'eau est souvent de bonne qualité et la pollution organique est restée faible. À travers son milieu aquatique relativement préservé ou une meilleure disponibilité en ressources trophiques ou encore une migration altitudinale des espèces en raison du réchauffement climatique, l'Oukaïmeden apparait comme une zone refuge pour la faune, le milieu présente un grand intérêt pour la conservation des espèces. En dépit de la régression du milieu aquatique constaté à l'Ourika, et de la diminution de la richesse spécifique qui en a découlé, l'habitat reste suffisamment adéquat pour permettre une recolonisation par un plus grand nombre d'espèces, à condition de limiter les perturbations, et que les activités anthropiques intègrent des mesures de préservation et de restauration de l'écosystème.

\section{REMERCIEMENTS}

Les auteurs remercient Mme Majida El Alami et les équipes du Laboratoire diversité et conservation des systèmes biologiques (LDICOSYB) de la faculté des sciences de Tétouan (Université Abdelmalek Essaadi, Maroc) pour l'aide précieuse apportée. 


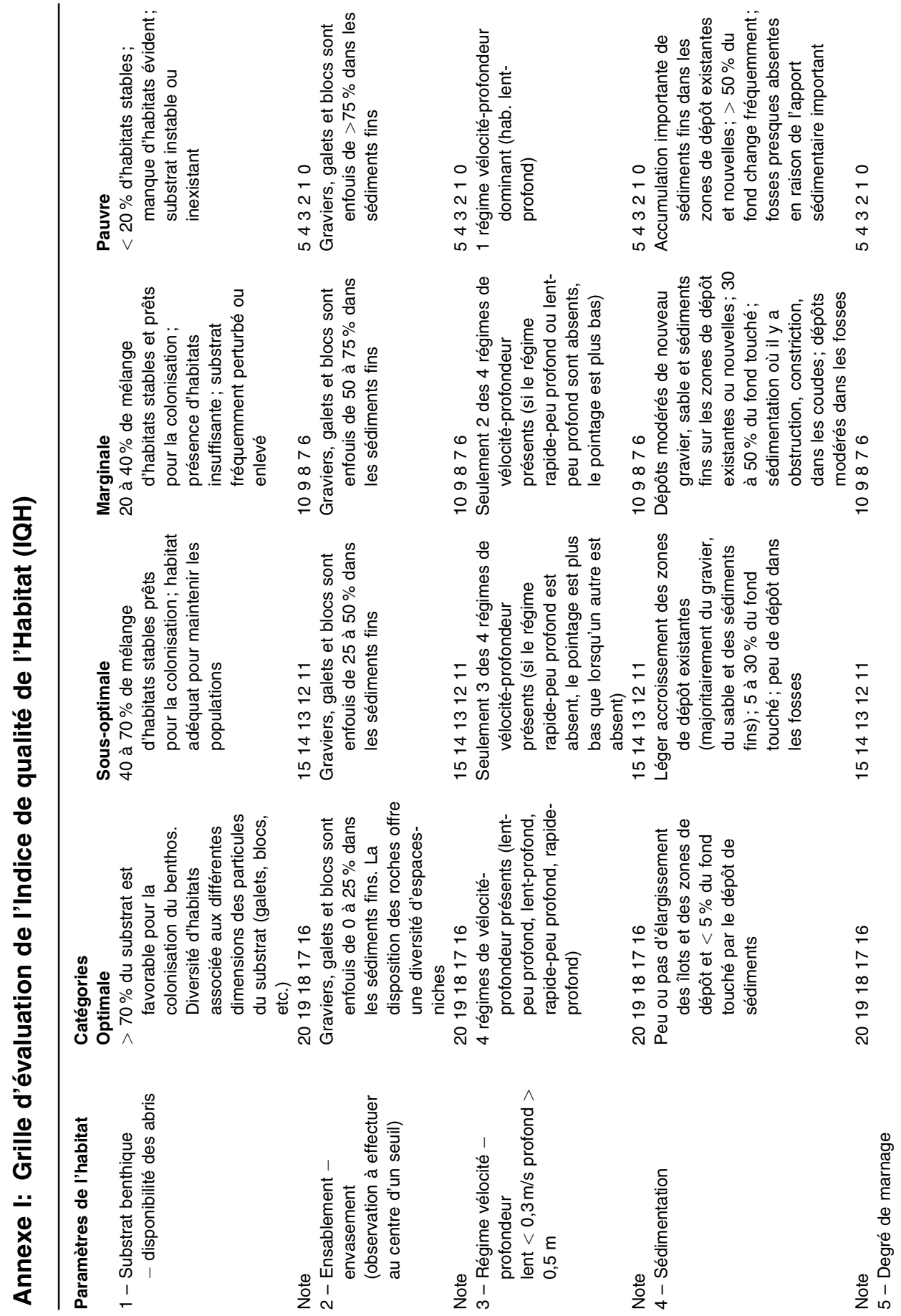


Impacts anthropiques et climatiques sur l'évolution des écosystèmes lotiques du Haut-Atlas marocain 143

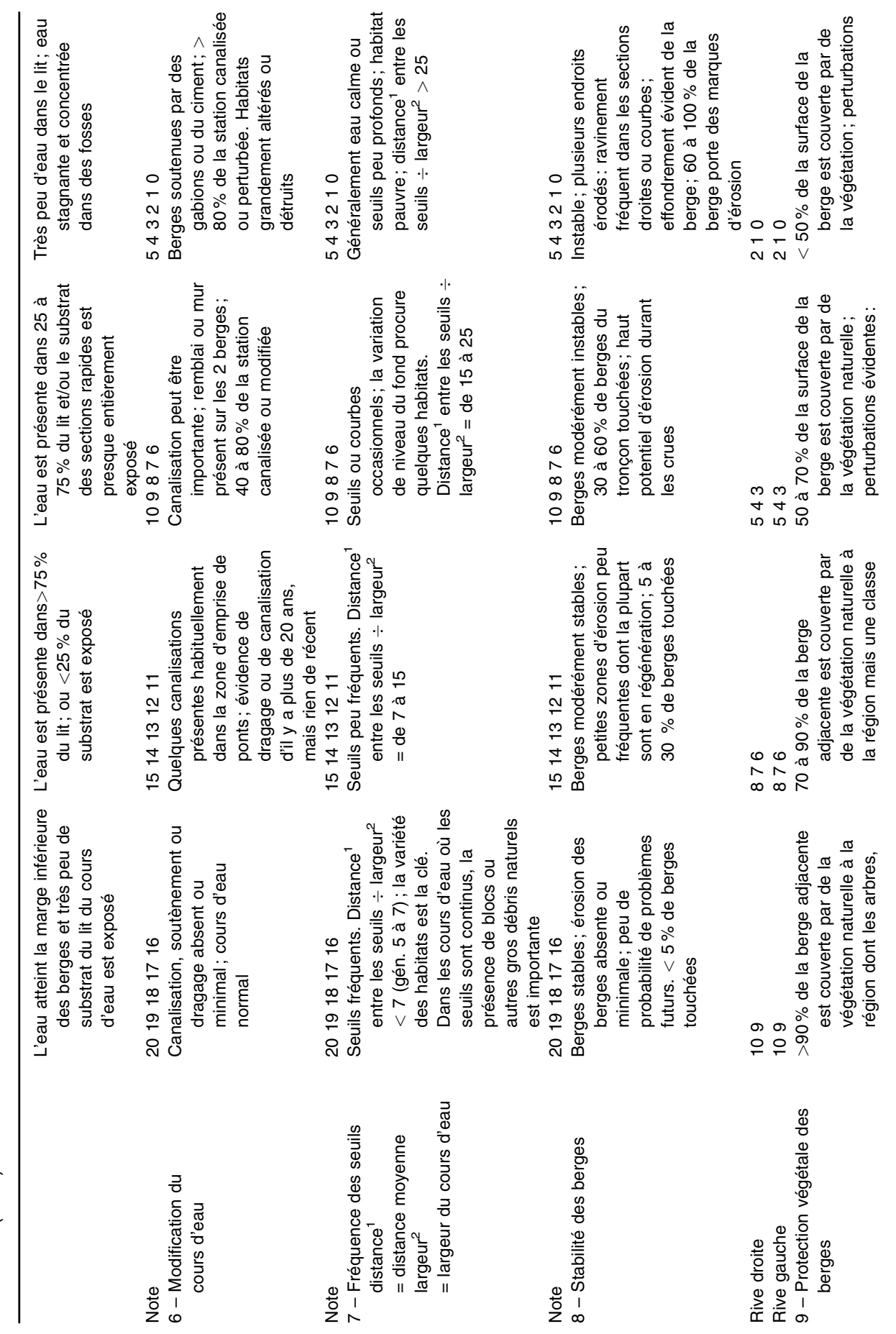




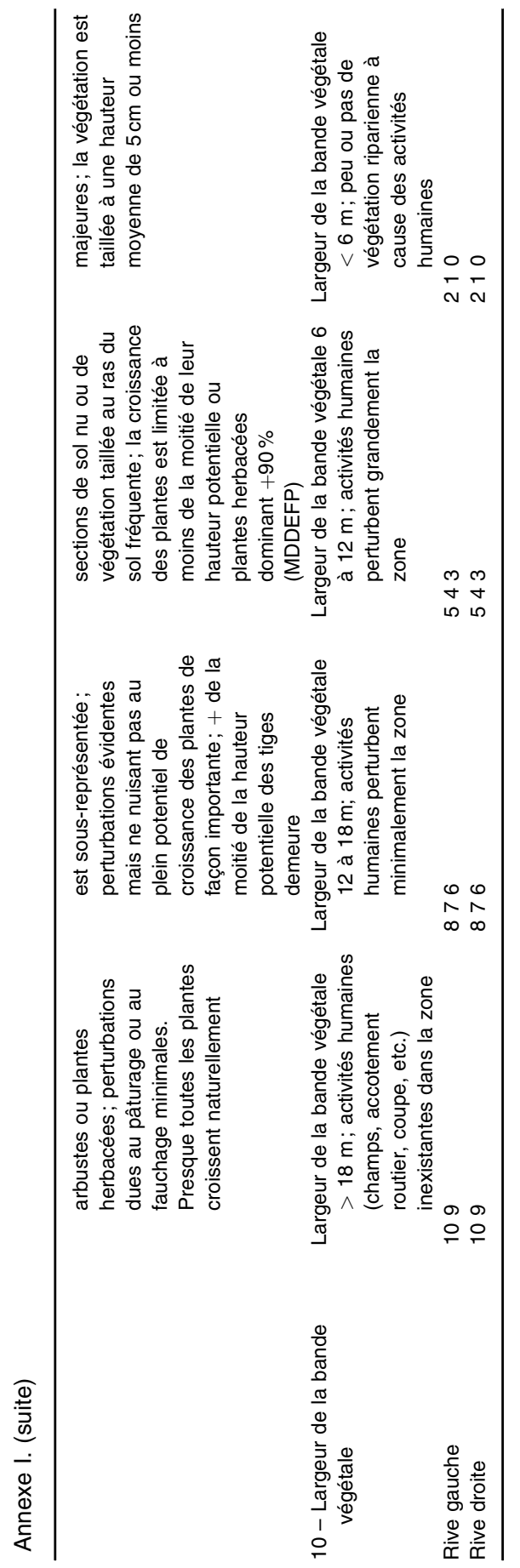




\section{Annexe II: Grille de calcul de I'IBGN.}

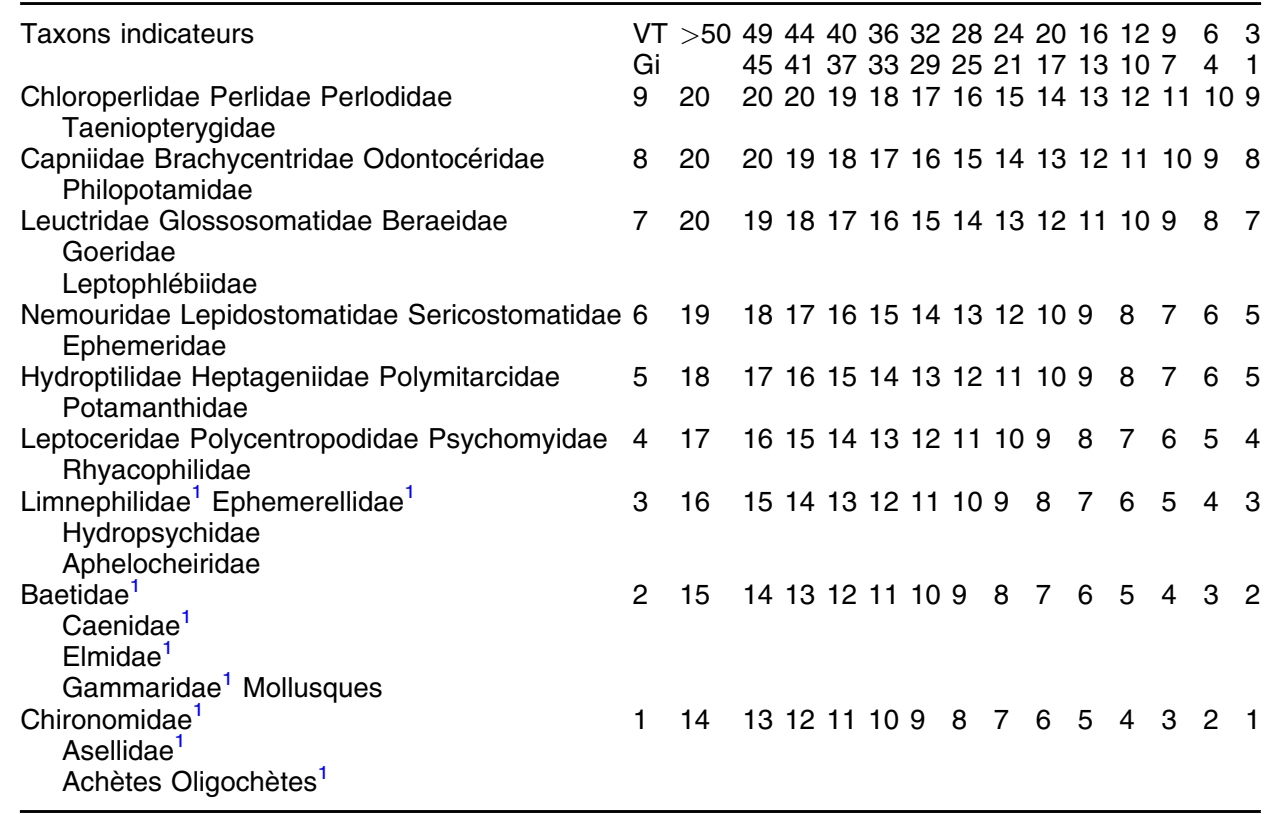

${ }^{1}$ Les taxa représentés par au moins 10 individus, les autres par au moins 3 individus.

\section{Annexe III: Inventaire des éphéméroptères}

\section{Baetidae}

Alainites muticus (Linné, 1758)

Matériel examiné. $01: 25$ août 2015, 1L; 16 octobre 2015, 3L. O2 : 6 janvier 2015, 3L; 13 mars 2015, 4L; 25 juillet 2015, 4L; 25 août 2015, 18L; 23 novembre 2015, 40L. O3: 6 janvier 2015, 55L; 10 mai 2015, 46L; 6 juin 2015, 12L; 25 août 2015, 169L.

Baetis gr. alpinus (Pictet, 1834), il s'agit très probablement de Baetis maurus (Kimmins, 1938) plusieurs fois recensée dans les stations.
Matériel examiné. OR1: 24 août 2015, 55L; 15 octobre 2015, 28L; OR2 : 24 août 2015, 2L; 30 décembre 2015, 6L. OR3 : 3 février 2015, 75L; 12 mars 2015, 4L; 24 août 2015, 4L; 15 octobre 2015, 32L. O1: 25 août 2015, 1L. O2: 25 juillet 2015, 20L; 25 août 2015, 30L; 16 octobre 2015, 50L; O3: 13 mars 2015, $1 \mathrm{~L}$; 25 juillet 2015, 53L; 25 août 2015, 60L.

Baetis pavidus (Grandi, 1949).

Matériel examiné. OR2:: 20 mai 2015, 14L; 22 juillet 2015, 22L; 24 août 2015, 2L. OR3 : 12 mars 2015, 2L ; 20 mai 2015, 126L; 11 juin 2015, 420L; 22 juillet 2015, 28L; 24 aoÛt 2015, 118L; 15 octobre 2015, 32L. O2: 25 juillet 2015, 4L; 25 août 2015, 2L. 


\section{Annexe III.}

\begin{tabular}{|c|c|c|c|c|c|c|c|c|c|c|c|c|}
\hline & \multicolumn{6}{|c|}{ Oukaïmeden } & \multicolumn{6}{|c|}{ Ourika } \\
\hline & \multicolumn{2}{|c|}{$\mathrm{O} 1$} & \multicolumn{2}{|c|}{$\mathrm{O} 2$} & \multicolumn{2}{|c|}{$\mathrm{O} 3$} & \multicolumn{2}{|c|}{ OR1 } & \multicolumn{2}{|c|}{ OR2 } & \multicolumn{2}{|c|}{ OR3 } \\
\hline & av & ap & av & ap & av & ap & av & ap & av & ap & av & ap \\
\hline \multicolumn{13}{|l|}{ Baetidae } \\
\hline Alainites muticus (Linné, 1758)* & - & + & - & + & - & + & - & - & - & - & - & - \\
\hline Baetis maurus (Kimmins, 1938) & + & & + & & + & & + & & + & & + & \\
\hline Alainites oukaïmeden (Thomas \& Sartori, 1992) & + & - & + & - & + & - & + & - & - & - & - & - \\
\hline Baetis rhodani (Pictet, 1843) & + & + & + & + & + & + & + & + & + & + & + & + \\
\hline Baetis gr. alpinus (Pictet, 1834). & & + & & + & & + & & + & & + & & + \\
\hline Baetis pavidus (Grandi, 1949) & - & - & - & + & - & - & + & - & + & + & + & + \\
\hline Cheleocholeon dimorphicum (Soldán \& Thomas, 1985) & - & - & - & - & - & - & + & - & - & - & - & - \\
\hline Cloeon dipterum (Linnaeus, 1761) & - & - & - & - & - & - & - & - & - & - & + & - \\
\hline $\begin{array}{l}\text { Nigrobeetis (Novikova \& Kluge, 1987)* } \\
\text { Oligoneuridae }\end{array}$ & - & - & - & + & - & - & - & - & - & - & - & + \\
\hline Oligoneuriella skoura (Dakki \& Giudicelli, 1980) & - & - & - & - & - & - & - & - & + & - & + & - \\
\hline $\begin{array}{l}\text { Oligoneuriopsis skhounate (Dakki \& Giudicelli, 1980) } \\
\text { Heptageniidae }\end{array}$ & - & - & - & - & - & - & - & - & - & - & + & - \\
\hline Ecdyonurus rotschildi (Navás, 1929) & + & + & + & + & - & + & + & + & + & + & + & + \\
\hline Epeorus sylvicola (Pictet, 1865) & + & + & + & + & + & + & + & + & + & - & + & + \\
\hline Rhitrogena (Eaton, 1881) & - & - & - & - & - & - & + & - & + & - & + & - \\
\hline \multicolumn{13}{|l|}{ Ephemerellidae } \\
\hline Serratella ignita (Poda, 1761) & + & + & + & + & + & + & + & + & - & + & + & + \\
\hline Caenidae & & & & & & & & + & & + & & \\
\hline Caenis pusilla (Navás, 1913) & + & + & + & + & + & + & - & & - & & - & + \\
\hline Caenis luctuosa (Bürmeister, 1839) & - & - & + & - & - & - & + & & + & & + & - \\
\hline \multicolumn{13}{|l|}{ Leptophlebiidae } \\
\hline Habroleptoides assafae (Sartori \& Thomas, 1986)* & - & + & - & + & - & + & - & - & - & - & - & - \\
\hline Habrophlebia (Eaton, 1881)* & - & + & - & + & - & + & - & - & - & - & - & - \\
\hline Choroterpes (Eaton, 1881) & - & - & - & - & - & + & - & - & - & - & - & - \\
\hline Nombre d'espèces Éphéméroptères & 8 & 9 & 8 & 11 & 6 & 10 & 10 & 6 & 8 & 6 & 11 & 8 \\
\hline
\end{tabular}

Baetis rhodani (Pictet, 1843)

Matériel examiné. O1: 5 janvier 2015, 180L; 2 février 2015, 504L; 13 mars 2015, 324L; 10 mai 2015, 674L; 6 juin 2015, 518L; 25 juillet 2015, 119L; 25 août 2015, 159L. O2: 6 janvier 2015, 2L; 2 février 2015, 3L; 13 mars 2015, $1 \mathrm{~L}$; 6 juin 2015, 115L; 25 juillet 2015, 1L; 25 août 2015, 74L; 16 octobre 2015, 150L; 23 novembre 2015, 3L. O3: 6 janvier 2015, 55L; 13 mars 2015, 344L; 10 mai 2015, 175L; 6 juin 2015, 12L; 25 juillet 2015, 52L; 25 août 2015, 244L. OR1: 7 janvier 2015, 8L ; 3 février 2015, 14L; 12 mars
2015, 62L; 20 mai 2015, 343L; 11 juin 2015, 282L; 22 juillet 2015, 38L; 24 août 2015, 44L; 15 octobre 2015, 28L. OR2 : 3 février 2015, 3L ; 12 mars 2015, 9L; 20 mai 2015, 1L; 22 juillet 2015, 45L; 24 août 2015, 1L; 30 décembre 2015, 3L. OR3 : 12 mars 2015, 17L; 20 mai $2015,6 \mathrm{~L} ; 11$ juin $2015,14 \mathrm{~L} ; 15$ octobre 2015, 30L.

Nigrobaetis sp. (Novikova \& Kluge, 1987).

Matériel examiné. OR3: 20 mai 2015, 27L. O2 : 6 janvier 2015, $1 \mathrm{~L}$.

Heptageniidae

Ecdyonurus rotschildi (Navás, 1929) 


\begin{tabular}{|c|c|c|c|c|c|c|c|c|c|c|c|c|}
\hline & \multicolumn{6}{|c|}{ Oukaïmeden } & \multicolumn{6}{|c|}{ Ourika } \\
\hline & \multicolumn{2}{|c|}{01} & \multicolumn{2}{|c|}{$\mathrm{O} 2$} & \multicolumn{2}{|c|}{ O3 } & \multicolumn{2}{|c|}{ OR1 } & \multicolumn{2}{|c|}{ OR2 } & \multicolumn{2}{|c|}{ OR3 } \\
\hline & av & ap & av & ap & av & ap & av & ap & av & ap & av & ap \\
\hline Nemouridae & & & & & & & & & & & & \\
\hline Amphinemoura chiffensis (Aubert, 1956) & + & + & + & - & + & + & + & - & - & - & - & - \\
\hline $\begin{array}{l}\text { Protonemura talboti (Navás, 1929) } \\
\text { Leuctridae }\end{array}$ & + & + & + & + & + & + & + & + & - & - & + & - \\
\hline $\begin{array}{l}\text { Leuctra maroccana (Aubert, 1956) } \\
\text { Capniidae }\end{array}$ & + & $\begin{array}{l}+ \\
+\end{array}$ & - & - & - & $\begin{array}{l}+ \\
+\end{array}$ & + & - & - & - & - & - \\
\hline $\begin{array}{l}\text { Capnia nigra (Pictet, 1833) } \\
\text { Perlidae }\end{array}$ & + & & - & - & - & & - & - & - & - & - & - \\
\hline Eoperla ochracea (Kolbe, 1885) & - & - & - & - & - & - & - & - & + & - & + & - \\
\hline Perla bipunctata (Pictet, 1833) & + & + & - & - & - & - & + & + & - & - & + & - \\
\hline Perla cf. pallida (Guérin-Méneville, 1838) & - & + & - & - & - & + & - & + & - & - & - & - \\
\hline $\begin{array}{l}\text { Perla marginata (Panzer, 1799) } \\
\text { Chloroperlidae }\end{array}$ & + & - & - & - & + & - & + & - & + & - & - & - \\
\hline $\begin{array}{l}\text { Siphonoperla lepineyi (Navás, 1935) } \\
\text { Perlodidae }\end{array}$ & + & + & - & $\begin{array}{l}- \\
+\end{array}$ & - & + & - & + & - & - & - & - \\
\hline Afroperlodes lecerfi (Navás, 1929) & + & + & - & & - & + & + & - & + & - & - & - \\
\hline Hemimelaena flaviventris (Pictet, 1841) & - & + & - & & - & + & - & + & - & - & - & - \\
\hline Taeniopterygidae (Klapálek, 1905) & - & - & - & - & - & + & - & - & - & - & - & - \\
\hline Nombre d'espèces Plécoptères & 8 & 9 & 2 & 2 & 3 & 9 & 6 & 5 & 3 & 0 & 3 & 0 \\
\hline
\end{tabular}

Matériel examiné. $01: 23$ novembre 2015, 13L. O2 : 23 novembre 2015, 5L. O3 : 6 janvier 2015, 1L ; 3 février 2015, $2 \mathrm{~L}$; 13 mars 2015, 15L; 25 juillet 2015, 4L; 25 août 2015, 51L; 15 octobre 2015, 20L; 23 novembre 2015, 23L. OR1: 24 août 2015, 1L; 15 octobre 2015, 4L. OR2: 20 mai 2015, 2L; 24 août $2015,1 \mathrm{~L} ; 15$ octobre $2015,14 \mathrm{~L}$; 30 décembre 2015, 3L. OR3: 20 mai 2015, 4L; 11 juin 2015, 3L; 22 juillet 2015, 3L ; 24 août 2015, 6L ; 15 octobre 2015, 6L.

Epeorus sylvicola (Pictet, 1865)

Matériel examiné. 01: 5 janvier 2015, 38L; 2 février 2015, 75L; 13 mars 2015, 30L; 10 mai 2015, 58L ; 6 juin 2015, 1L; 25 juillet 2015, 1L; 25 août 2015, 17L; 16 octobre 2015, 5L. O2: 25 août 2015, 2L; 16 octobre 2015, 14L. 23 novembre 2015, 25L. O3 : 6 janvier 2015, 2L ; 3 février 2015,
4L; 13 mars 2015, 14L; 10 mai 2015, 23L; 06 juin 2015, 8L; 25 juillet 2015, 6L; 25 août 2015, 83L; 16 octobre 2015, 10L; 23 novembre 2015, 30L. OR1 : 7 janvier 2015, $1 \mathrm{~L}$; 24 août 2015, 4L. OR3: 15 octobre 2015, $1 \mathrm{~L}$.

\section{Ephemerellidae}

Serratella ignita (Poda, 1761)

Matériel examiné. 01: 2 février 2015, 2L; 10 mai 2015, 3L; 6 juin 2015, 168L; 25 juillet 2015, 23L ; 16 octobre 2015, 3L. O2 : 6 juin 2015, 37L; 25 juillet 2015, 56L; 23 novembre 2015, 2L; O3: 10 mai 2015, 10L; 6 juin 2015, 38L; 25 juillet 2015, $1 \mathrm{~L}$. OR1 : 12 mars 2015, 1L; 20 mai 2015, 26L ; 11 juin 2015 , 45L ; 22 juillet 2015 , 3L. OR2: 12 mars 2015, 1L; 20 mai 2015, 3L; 11 juin 2015, 8L. OR3: 12 mars 2015, 1L; 20 mai 2015, 7L; 11 juin 2015, $2 \mathrm{~L}$.

Caenidae 


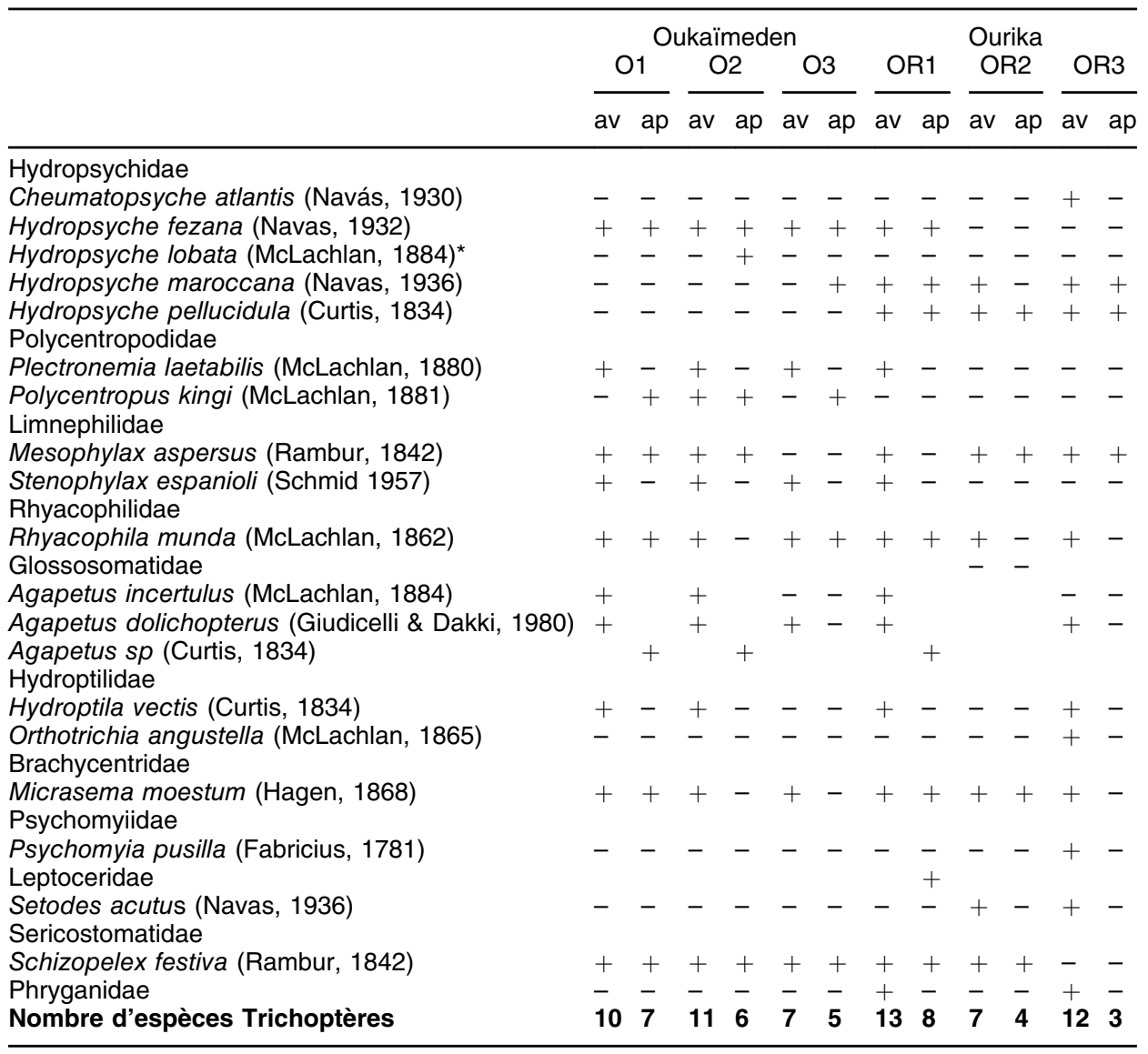

Matériel examiné. OR1: 22 juillet 2015, 1L. OR2 : 22 juillet 2015, 1L ; 24 août 2015, 2L; 15 octobre 2015, 2 L.

Caenis pusilla (Navás, 1913)

Matériel examiné. OR3: 20 mai 2015, 2L; 22 juillet 2015, 1L. O1: 5 janvier 2015, 1L; 2 février 2015, 25L; 10 mai 2015, 7L; 6 juin 2015, 11L; 23 novembre 2015, 57L. O2: 16 octobre 2015, 3L; 23 novembre 2015, 5L. O3: 6 janvier 2015, 19L; 3 février 2015, 22L ; 13 mars 2015, 35L; 10 mai 2015, 121L ; 6 juin 2015, 35L ; 25 août 2015, $3 \mathrm{~L}$; 16 octobre 2015, 3L.

\section{Leptophlebiidae}

Habroleptoides assefae (Sartori \& Thomas, 1986).

Matériel examiné. 01: 10 mai 2015, 1L; 24 juillet 2015, 4L; 25 août 2015, 1L; 23 novembre 2015, 11L. O2: 23 novembre 2015, 7L. O3:: 6 janvier 2015, 7L; 6 juin 2015, 6L; 15 octobre 2015, 10L; 23 novembre 2015, 5L.

Habrophlebia sp. (Eaton, 1881)

Matériel examiné. O1: 25 juillet 2015, 04L; 25 août 2015, 1L. O2: 25 août 2015, 2L. O3 : 6 janvier 2015, $1 \mathrm{~L}$; 25 août 2015, 6L. 
Choroterpes sp. (Eaton, 1881).

Matériel examiné. O3: 13 mars 2015, 6L; 10 mai 2015, 6L; 25 juillet 2015, 3L; 23 novembre 2015, 27L.

\section{Annexe IV: Inventaire des plécoptères.}

\section{Capniidae}

Capnia sp (Pictet, 1841).

Matériel examiné. $01: 5$ janvier 2015, 8L. O3: 5 janvier 2015, 1 L.

Chloroperlidae

Siphonoperla lepineyi (Navás, 1935)

Matériel examiné. 01 : 2 février 2015, 11L; 13 mars 2015, 4L; 10 mai 2015, 52L. O3: 13 mars 2015, 22L ; 21 mai 2014, 4L.

Leuctridae

Leuctra maroccana (Aubert, 1956)

Matériel examiné. O1: 13 mars 2015, 1L; 10 mai 2015, 1L. O3: 6 janvier 2015, 1L.

Nemouridae

Amphinemoura chiffensis (Aubert, 1956)

Matériel examiné. 01: 5 janvier 2015, 17L; 2 février 2015, 18L; 13 mars 2015, 2L; 25 juillet 2015, 7L; 25 août 2015, 5L; 23 novembre 2015, 145L. O3: 6 janvier 2015, 4L ; 3 février 2015, 4L; 13 mars 2015, 6L; 25 août 2015, 7L; 16 octobre 2015, 5L; 23 novembre 2015, $20 \mathrm{~L}$.

Protonemura talboti (Navás, 1929)

Matériel examiné. O1: 5 janvier 2015, 6L; 2 février 2015, 5L. O2: 25 juillet 2015, 1L. O3 : 6 janvier 2015, 1L; 3 février 2015, 62L; 13 mars 2015, 1L;

Perlidae

Perla bipunctata (Pictet, 1833)
Matériel examiné. 01: 2 février 2015, 1L; 13 mars 2015, 1L; 10 mai 2015, 1L; 25 juillet 2015, 4L. OR1 : 24 août 2015, 1L; 15 octobre 2015, $1 \mathrm{~L}$.

Perla cf. pallida (Guérin-Méneville, 1838). Les spécimens de Perla marginata (Panzer, 1799) cité dans le HautAtlas (Pihan \& Mohati 1983; Bouzidi 1989) ont été assignés à $P$. cf. pallida (Mabrouki et al., 2016).

Matériel examiné. 01: 10 mai 2015, $1 \mathrm{~L}$; 25 juillet 2015, 2L; 16 octobre 2015, 6L. O3: 6 janvier 2015, 1L; 3 février 2015, 2L; 6 juin 2015, 1L; 25 juillet $2015,1 \mathrm{~L}$. OR1 : 20 mai $2015,1 \mathrm{~L}$; 11 juin 2015, 4L ; 22 juillet 2015, 3L.

Perlodidae

Afroperlodes lecerfi (Navás, 1929)

Matériel examiné. O1: 2 février 2015, 1L; 10 mai 2015, 7L. O3: 13 mars 2015, 1L; 6 juin 2015, 5L.

Hemimelaena flaviventris (Pictet, 1841)

Matériel examiné. 01 : 10 mai 2015, 8L; 6 juin 2015, 11L. O3: 13 mars 2015, 3L; 10 mai 2015, 47L; 6 juin 2015, 6L. OR1 : 12 mars 2015, 1L; 20 mai $2015,1 \mathrm{~L}$.

Taeniopterygidae.

Matériel examiné. O3: 6 janvier 2015, 2L.

\section{Annexe V: Inventaire des trichoptères}

\section{Brachycentridae}

Micrasema moestum (Hagen, 1868)

Matériel examiné. O1: 5 janvier 2015, 3L. OR1: 7 janvier 2015, 4L; 20 mai 2015, 3L. OR2 : 7 janvier 2015, 3L ; 3 février 2015, 2L. 
Glossosomatidae

Agapetus sp (Curtis, 1834). Nous pensons que Agapetus incertulus (McLachlan, 1884) et Agapetus dolichopterus (Giudicelli \& Dakki, 1980) autrefois recensées sont toujours présentes, les spécimens du genre Agapetus recensés au cours de nos prospections non pas pu être identifiés au niveau spécifique en raison du stade juvénile de larves.

Matériel examiné. 01: 6 juin 2015, 3L. OR1 : 7 janvier 2015, 1L; 3 février 2015, 1 L.

Hydropsychidae

Hydropsyche fezana (Navas, 1932)

Matériel examiné. O1: 25 juillet 2015, 41L. O2: 15 octobre 2015, 25L. O3: 13 mars 2015, 20L. OR1: 12 mars 2015, 3L.

Hydropsyche lobata (McLachlan, 1884).

Matériel examiné. O2: 16 octobre 2015, 24L.

Hydropsyche maroccana (Navas, 1936)

Matériel examiné. O3: 13 mars 2015. 8L. OR1: 20 mai $2015,1 \mathrm{~L}$. OR3: 12 mars 2015, 4L.

Hydropsyche pellucidula (Curti, 1834)

Matériel examiné. O2: 16 octobre 2015, 2L. OR1: 15 octobre 2015, 7L. OR2: 24 août 2015, 6L. OR3: 11 juin 2015, 2 L.

Limnephilidae

Mesophylax aspersus (Rambur, 1842)

Matériel examiné. 01: 5 janvier 2015, 3L; 6 juin 2015, 2L. OR2: 03 janvier 2015, 10L. OR3 : 3 février 2015, 1L; 12 mars 2015, $1 \mathrm{~L}$.

Polycentropodidae
Polycentropus kingi (McLachlan, 1881)

Matériel examiné. 01: 2 février 2015, 1L. O2: 6 janvier 2015, 3L ; 2 février 2015, 6L; 1 mars 2015, 3L; 25 juillet 2015, 49L ; 25 août 2015, 26L ; 16 octobre 2015, 21L; 23 novembre 2015, 9L. O3: 6 janvier 2015, 1L; 25 juillet 2015, 2L.

Leptoceridae

Matériel examiné. OR1: 7 janvier 2015, 3L.

Rhyacophilidae

Rhyacophila munda (McLachlan, 1862)

Matériel examiné. 01: 5 janvier 2015, 3L. O3: 10 mai 2015, 1L; 6 juin 2015, 7L; 25 juillet 2015, 4L ; 25 août 2015, 11L; 16 octobre 2015, 4L; 23 novembre 2015, 9L. OR1: 7 janvier 2015, 1L; 3 février 2015, $1 \mathrm{~L}$.

Sericostomatidae

Schizopelex festiva (Rambur, 1842)

Matériel examiné. $01: 5$ janvier 2015, 12L ; 2 février 2015, 45L ; 13 mars 2015, 21L; 10 mai 2015, 10L; 6 juin 2015, 12L; 23 novembre 2015, 8L. O2: 6 janvier 2015, 1L; 13 mars 2015, 42L; 23 novembre 2015, 1L. O3: 6 janvier 2015, 7L; 0 février 2015, 6L; 10 mai 2015, 2L ; 25 août 2015, 12L; 16 octobre 2015, 1L; OR1: 7 janvier 2015 1L; 20 mai 2015, 14L. OR2 : 7 janvier 2015, 1 L.

\section{RÉFÉRENCES}

Ajakane A. 1988. Étude hydrobiologique du bassin versant de l'oued N'fis (Haut Atlas marocain)- biotypologie, dynamique saisonnière, impact de l'assèchement sur les communautés benthiques. Thèse Doctorat. Marrakech: Univ. Cadi Ayyad, 189 p. 
Alaoui A. 2006. Trichoptères du Bassin de Oued Laou et la région de Fifi (Rif occidental). Thèse de $3^{\mathrm{e}}$ cycle. Tétouan: Univ. Abdelmalek Essaadi, $84 \mathrm{p}$.

Aubert J. 1956. Contribution à l'étude des Plécoptères d'Afrique du Nord. Mitt. Schweiz. Entomol. Ges. 29: 419-436.

Ayt Ougougdal H., Yacoubi Khebiza M., Messouli M., Lachir A. 2020. Assessment of Future Water Demand and Supply under IPCC Climate Change and Socio-Economic Scenarios, Using a Combination of Models in Ourika Watershed, High Atlas, Morocco. Water 12(6): 1751. https://doi.org/10.3390/ w12061751.

Badri A. 1985. Étude hydrobiologique d'un cours d'eau de plaine en zone semiaride: le Tensift. Impact des crues sur la biocénose. Thèse de $3^{e}$ cycle. Marrakech: Univ. Cadi Ayyad, 192 p.

Badri A. 1993. Influence des crues sur les écosystèmes lotiques du Haut-Atlas: Etude des perturbations et des mécanismes de recolonisation à travers les peuplements des algues et des invertébrés. Thèse d'État. Maroc: Univ. Cadi Ayyad, 192 p.

Barbour M.T., Gerritsen J., Snyder B.D. \& Stribling J.B. 1999. Rapid Bioassessment Protocols for Use in Wadeable Rivers: Periphyton, Benthic Macroinvertebrates, and Fish, $2^{\mathrm{e}}$ édition, Washington, D.C.: U.S. Environmental Protection Agency, Office of Water, EPA841-B-99-002, 11 chapitres, 4 annexes. http://www.epa.gov/owow/ monitoring/rbp/wp61pdf/rbp.pdf.

Bebba N., El Alami M., Arigue S.F. \& Arab A. 2015. Étude mésologique et biotypologique du peuplement des Éphéméroptères de l'oued Abdi (Algérie). JMES (6): 1164-1177.
Berrahou A., Cellot B. \& Richoux P. 2001. Distribution des macroinvertébrés benthiques de la Moulouya et de ses principaux affluents (Maroc). Ann. Limnol. 37(3): 223-235.

Bonada N., Zamora-Muñoz C., El Alami M., Múrria C. \& Prat N. 2008. New records of Trichoptera in reference mediterranean climate rivers of the Iberian Peninsula and north of Africa: $T$ axonomical, Faunistical and Ecological aspects. Graellsia 64(2): 189-208.

Bode R.W., Novak M.A. \& Abele L.E. 1991. Quality assurance work plan for biological stream monitoring in New York State. Albany, New York: NYS Department of Environmental Conservation, Division of water, $80 \mathrm{p}$.

Botosaneanu L. 1975. Trichoptères recueillis au Maroc par M.I. Miron. Folia ent. hung. S.N. 28(2): 269-276.

Bouzidi A. 1989. Recherches hydrobiologiques sur les cours d'eau des massifs du Haut- Atlas (Maroc). Bio-écologie des macroinvertébrés et distribution spatiale des peuplements. Thèse Doctorat d'Etat. Marrakech: Univ. Cadi Ayyad, $190 \mathrm{p}$.

Cayrou J., Compin A., Giani N. \& Cereghino R. 2000. Associations spécifiques chez les macroinvertébrés benthiques et leur utilisation pour la classification des cours d'eau. Cas du réseau hydrographique Adour-Garonne (France). Ann. Limnol. (36): 189-202.

Croyle B.T. 1997. Population and community ecology of stream macroinvertebrates: The role of disturbance. A thesis in zoology of the Requirements for the Degree of Master of Science, Graduate Faculty of Texas Tech University, $77 \mathrm{p}$.

Cummins K.W. 1975. Macroinvertebrates. In: River Ecology, Studies in Ecology (B.A. Witthon, Ed.), pp. 170-198. 
Berkeley (Californie): University of California Press, 2.

Dakki M. 1978. Le genre Hydropsyche au Maroc (Trichoptera Hydropsychidae). Bulletin de I'Institut Scientifique. Rabat 3: 111-119.

Dakki M. 1982. Trichoptères du Maroc. Bulletin de l'Institut Scientifique, Rabat 6: 139-155

Dakki M. 1986. Recherches hydrobiologiques sur le haut Sebou (Moyen Atlas); une contribution à la connaissance faunistique, écologique et historique des eaux courantes Sud-rnéditerranéennes. Thèse es. Science. Rabat, $181 \mathrm{p}$.

Dakki M. 1987. Ecosystème d'eau courante du haut Sebou (Moyen Atlas); Etudes typologiques et analyses écologiques et biogéographie des principaux peuplements entomologiques. Travaux de L'Institut Scientifique. Série Zoologie 42: 99.

Dunn S.M., Brown I., Sample J., Post H. 2012. Relationships between climate, water resources, land use and diffuse pollution and the significance of uncertainty in climate change. J. Hydrol. 434: 19-35.

El Agbani M.A. 1984. Le réseau hydrographique du bassin versant de l'oued Bou Regreg (plateau central marocain). Essai de biotypologie. Thèse de $3^{\mathrm{e}}$ cycle. Univ. Claude Bernard, Lyon I. 147 p.

El Alami M. \& Dakki M. 1998. Peuplements d'Ephéméroptères et de Trichoptères de l'Oued Laou (Rif Occidentale, Maroc): distribution longitudinale et biotypologie. Bulletin de l'Institut Scientifique de Rabat 21: 51.

El Alami M. 2002. Taxonomie, écologie et biogéographie des Éphéméroptères du Rif (Nord du Maroc). Thèse de doctorat d'Etat Hydrobiologie. Maroc: Univ. Abdelmalek Essaadi, Fac. Sci. Tétouan.

El Alami M., Dakki M., Errami M. \& AlbaTercedor J. 2000. Nouvelles données sur les Baetidae du Maroc (Insecta, Ephemeroptera). Zool. Baetica 11: 105-113.

El Bazi R., El Alami M., Khadri O., Errochdi S., Slimani M. \& Bennas N. (2017). Projet du parc naturel de Bouhachem (Nord-Ouest du Maroc) II: Ephemeroptera, Plecoptera, Trichoptera. Boletín de la Sociedad Entomológica Aragonesa (S.E.A.) 61: 55-66.

Errochdi S., El Alami M., Vinçon G., Abdaoui A. \& Ghamizi M. 2014. Contribution to the knowledge of Moroccan and Maghrebin stoneflies (Plecoptera). Zootaxa 3838(1): 46-76. ;1;.

Floury M., Delattre C., Ormerod S.J., Souchon Y. 2012. Global versus local change effects on a large European river. Sci. Total Environ. 441: 220-229.

Giudicelli J., Bouzidi A. \& Ait Abdelaali N. 2000. Contribution à l'étude faunistique et écologique des simulies (Diptèra: Simuliidae) du Maroc IV. Les simulies du Haut-Atlas. Description d'une nouvelle espèce. Ann. Limnol. - Int. J. Lim. 36 (1): 57-80. https://doi.org/10.1051/ $\operatorname{limn} / 2000005$.

Giudicelli J. \& Dakki M. 1984. Les sources du Moyen Atlas et du Rif (Maroc): Faunistique (description de deux espèces nouvelles de Trichoptères), écologie, intérêt biogéographique Bijd. Dierkunde 54(1): 83-100.

Hajji, K., Zamora-Muñoz C., Bonada N. \& El Alami M. 2012. Quelques notes sur l'écologie et distribution des Rhyacophilidae du Rif (du Maroc). Boletín de la S.E.A. 50: 559-562. 
Hajji K., El Alami M., Bonada N. \& ZamoraMuñoz C. 2013. Contribution à la connaissance des Trichoptères (Trichoptera) du Rif (Nord du Maroc). Boln. Asoc. esp. Ent. 37(3-4): 181-216.

Hajji K. 2017. Les Trichoptères du Maroc: Atlas, Biogéographie et degré de vulnérabilité. Thèse Doctorat d'État. Tétouan: Univ. Abdelmalek Essaâdi, $309 \mathrm{p}$.

Hellawell J.M. 1986. Biological Indicators of Freshwater Pollution and Environmental Management. Londres: Elsevier, $546 \mathrm{p}$.

Hilsenhoff W.L. 1988. Rapid field assessment of organic pollution with familylevel biotic index. J. N. Am. Benthol. Soc. 7(1): 65-68.

Hynes, H.B.N. 1970. The Ecology of Running Waters. Toronto: University of Toronto, $555 \mathrm{p}$.

Iliopoulou-Georgudaki J., Kantzaris V., Katharios P., Kaspiris P., Georgiadis T. \& Montesantou B. 2003. An application of different bioindicators for assessing water quality: a case study in the rivers Alfeios and Pineios (Peloponnisos, Greece). Ecol. Indic. 2: 345360.

Klemm D.J., Lewis P.A., Fulk F. \& Lazorchak J.M. 1990. Macroinvertebrate field and laboratory methods for evaluating the biological integrity of surface waters. Cincinnati, Ohio: U.S. Environmental Protection Agency, Office of Research and Development, Environmental monitoring systems laboratory, EPA/600/4-90/030, $256 \mathrm{p}$.

Leclercq L. \& Maquet B. 1987. Deux nouveaux indices chimiques et diatomiques de la qualité d'eau courante. Application au Samson et à ses affluents (bassin de la Meuse Belge). Comparaison avec d'autres indices chimiques, biologiques et diatomiques.
Institut Royal des Sciences Naturelles de Belgique. Documents de travail. No38, $98 \mathrm{p}$.

Lenat D.R. 1988. Water quality assessment of streams using a qualitative collection method for benthic macroinvertebrates. J. N. Am. Benthol. Soc. 7: 222-233.

Mabrouki Y., Taybi A.F., Chavanon G., Vinçon G. \& Berrahou A. 2016. Contribution à l'étude des plécoptères dans le Maroc Oriental et le bassin versant de la Moulouya et leur distribution en fonction des étages bioclimatiques. J. Mater. Environ. Sci. 7(6): 2178-2193

Ministère du Développement durable, de l'Environnement, de la Faune et des Parcs (MDDEFP). 2013. Guide de surveillance biologique basée sur les macroinvertébrés benthiques d'eau douce du Québec - Cours d'eau peu profonds à substrat grossier, 2013. Direction du suivi de l'état de l'environnement, ISBN 978-2-550-69169-3 (PDF), $2^{\mathrm{e}}$ édition, 88 p. (incluant 6 ann.).

Mohati A. 1985. Recherches hydrobiologiques sur un cours d'eau du Haut Atlas de Marrakech (Maroc): L'oued Ourika, Ecologie, biotypologie et impacts des activités humaines sur la qualité des eaux. Thèse de $3^{\mathrm{e}}$ cycle. Marrakech: Univ. Cadi Ayyad, 108 p.

Mohseni O., Stefan H.G. 1999. Stream temperature/air temperature relationship: a physical interpretation. J. Hydrol. 218: 128-141.

Moisan J. \& Pelletier L. 2008. Guide de surveillance biologique basée sur les macroinvertébrés benthiques d'eau douce du Québec - Cours d'eau peu profonds à substrat grossier, 2008. Direction du suivi de l'état de l'environnement, ministère du Développement durable, de l'Environnement et des Parcs, ISBN: 978-2-550-53591-1 (version imprimée), 86 p. (incluant 6 ann.). 
Mosely M.E. 1938. Trichoptera collected in Morocco by Mrs. K.H. Chapman and G. A. Bisset. Ann. Mag. Nat. Hist. London 2(1): 271-277.

Ouahsine H. 1993. Les Biocénoses d'Invertébrés benthiques dans un torrent du Haut Atlas (Maroc): Le Tiferguine. Structure et répartition du peuplement - Régime alimentaire, Dynamique des populations et production des espèces dominantes. Thèse Doctorat d'Etat. Marrakech: Univ. Cadi Ayyad, 234 p.

Parsons M. \& Norris R.H., 1996. The effect of habitat specific sampling on biological assessment of water quality using a predictive model. Freshwater Biol. 36: 419-434.

Pihan J-C. \& Mohati A. 1983. Étude hydrobiologique de deux petits torrents du haut Atlas de Marrakech L'assif Tiferguine et l'assif Oukaïmeden. Impact des activités humaines. Bulletin de la Faculté des Sciences de Marrakech (Section Sciences de la Vie) 2: 23-61.

Plafkin J. L., Barbour M.T., Porter K.D., Gross S.K. \& Hughes R.M. 1989. Rapid Bioassessment Protocols for use in Streams and Rivers: Benthic Macroinvertebrates and Fish. U.S. Environmental Protection Agency. EPA 440/489/001. 8 chapters, appendices A-D.

Resh V.H., Norris R.H. \& Barbour M.T. 1995. Design and implementation of rapid assessment approaches for water resource monitoring using benthic macroinvertebrates. Aust. J. Ecol. 20 : 108-121.

Resh V.H., Myers, M.J. \& Hannaford M.J. 1996. Macroinvertebrates as biotic indicators of environmental quality. In: Methods in Stream Ecology, pp. 647667. Academic Press.

Saidi M.E.M., Daoudi L., El hassane Aresmouk M., Blali, A. 2003. Rôle du milieu physique dans l'amplification des crues en milieu montagnard : exemple de la crue du 17 août 1995 dans la vallée de l'Ourika (Haut-Atlas, Maroc). Science et changements planétaires/ Sécheresse 14(2) : 107-114.

Saidi El Mehdi M., Daoudi L., Aresmouk M., Fniguire F., Boukrim S. 2010. Les crues de l'oued Ourika (Haut Atlas, Maroc): Événements extrêmes en contexte montagnard semi-aride. Comunicações Geológicas 97: 113-128.

Sánchez-Ortega A., Alba-Tercedor J., Tierno de Figueroa J.M. 2003. Lista faunística y bibliográfica de los Plecópteros de la Península Ibérica de Islas Baleares. Publicaciones de la Asociación espaňola de Limnología 16: 198.

Scrimgeour, G.J., Prowse T.D., Culp J.M. \& Chambers P.A. 1994. Ecological effects of river ice break-up: a review and perspective. Freshw. Biol. 32: 261-275.

Soldner M., Stephen I., Ramos L., Angus R., Claire-Wells N., Grosso A. \& Crane M. 2004. Relationship between macroinvertebrate fauna and environmental variables in small streams of the Dominican Republic. Water Res. 38: 863-874.

Talami Z. 1998. Application des méthodes biologiques et chimiques pour la détermination de la qualité des eaux des rivières du Haut-Atlas marocain. Cas des oueds Tensift, Oukaimeden et Ourika. Thèse Hydrobiologie. Marrakech: Univ. Cadi Ayyad, 221 p.

Tayoub H. 1989. Étude hydrobiologique d'un réseau hydrographique rifain, l'oued Laou: Typologie et Écologie des trichoptères. Thèse Doctorat 3ème cycle, Faculté des Sciences de Rabat, $137 \mathrm{p}$.

Thomas A. \& Bouzidi A. 1986. Trois Ephéméroptères nouveaux du Haut Atlas marocain (Heptageniidae, Baetidae, Leptophlebiidae). Bull. Soc. Hist. Nat., Toulouse 122: 7-10.

Thomas, A., Bouzidi, A., Sartori, M., Assef, S. \& Ajakane A. 1992. Compléments et 
corrections à la faune des Ephéméroptères d'Afrique du Nord. 5. Baetis oukaimeden n. sp. du Haut Atlas marocain: description et écologie (Ephemeroptera, Baetidae). Mitt. Schweiz. entomol. Ges. 65: 369-377.

Townsend C.R., Scarsbrook M.R. \& Dolédec S. 1997. The intermediate disturbance hypothesis, refugia, and biodiversity in streams. Limnol. Oceanogr. 42(5): 938-949.

Tuffery, G., (1980). Incidences écologiques de la pollution des eaux courantes, révélateurs biologiques de la pollution. In: La pollution des eaux continentales. Incidence sur les biocénoses aquatiques (P. Pesson, Ed.), pp. 243-280. Gauthier-Villars.

United States Environmental Protection Agency (U.S. EPA). 2002. Biological
Assessments and Criteria: Crucial Components of Water Quality Programs. United States Environmental Protection, Agency, EPA822-F-02006. http://www.epa.gov/ost/biocriteria/ technical/brochure.pdf.

Vinçon, G., El Alami M. \& Errochdi S. 2014. Contribution to the knowledge of the Moroccan High and Middle Atlas stoneflies (Plecoptera, Insecta). Illiesia 10(3): 17.

Water Framework Directive (WFD), 2003. Overall Approach to the Classification of Ecological Status and Ecological Potential, Water Framework Directive Common Implementation Strategy Working Group 2, A Ecological Status (ECOSTAT), 28 p. http://cwss.www.de/workshops/ wfd/Ecological-Classification-Gui dance.pdf. 